\title{
Shape preserving design of geometrically nonlinear structures using topology optimization
}

Li, Yu; Zhu, Jihong ; Wang, Fengwen; Zhang, Weihong ; Sigmund, Ole

Published in:

Structural and Multidisciplinary Optimization

Link to article, DOI:

$10.1007 / \mathrm{s} 00158-018-2186-\mathrm{x}$

Publication date:

2019

Document Version

Peer reviewed version

Link back to DTU Orbit

Citation $(A P A)$ :

Li, Y., Zhu, J., Wang, F., Zhang, W., \& Sigmund, O. (2019). Shape preserving design of geometrically nonlinear structures using topology optimization. Structural and Multidisciplinary Optimization, 59(4), 1033-1051. https://doi.org/10.1007/s00158-018-2186-x

\section{General rights}

Copyright and moral rights for the publications made accessible in the public portal are retained by the authors and/or other copyright owners and it is a condition of accessing publications that users recognise and abide by the legal requirements associated with these rights.

- Users may download and print one copy of any publication from the public portal for the purpose of private study or research.

- You may not further distribute the material or use it for any profit-making activity or commercial gain

- You may freely distribute the URL identifying the publication in the public portal 


\title{
Shape preserving design of geometrically nonlinear structures using topology optimization
}

\author{
Yu Li ${ }^{\mathrm{a}, *}$, Jihong Zhu ${ }^{\mathrm{a}, \mathrm{b}, *}$, Fengwen Wang ${ }^{\mathrm{c}}$, Weihong Zhang ${ }^{\mathrm{a}}$, Ole Sigmund ${ }^{\mathrm{c}}$ \\ ${ }^{a}$ State IJR Center of Aerospace Design and Additive Manufacturing \\ Northwestern Polytechnical University, Xian, Shaanxi 710072, China \\ ${ }^{b}$ MIIT Lab of Metal Additive Manufacturing and Innovative Design \\ Northwestern Polytechnical University, Xian, Shaanxi 710072, China \\ ${ }^{c}$ Department of Mechanical Engineering, Section for Solid Mechanics \\ Technical University of Denmark, DK-2800 Lyngby, Denmark
}

\begin{abstract}
Subparts of load carrying structures like airplane windows or doors must be isolated from distortions and hence structural optimization needs to take such shape preserving constraints into account. The paper extends the shape preserving topology optimization approach from simple linear load cases into geometrically nonlinear problems with practical significance. Based on an integrated deformation energy function, an improved warpage formulation is proposed to measure the geometrical distortion during large deformations. Structural complementary elastic work is assigned as the objective function. The average distortion calculated as the integrated deformation energy accumulated in the incremental loading process is accordingly constrained to obtain warpage control. In the numerical implementation, an energy interpolation scheme is utilized to alleviate numerical instability in low stiffness regions. An additional loading case avoids isolation phenomena. Optimization results show that shape preserving design is successfully implemented in geometrically nonlinear structures by effectively suppressing local warping deformations.
\end{abstract}

Keywords: Shape preserving design, Topology optimization, Geometrical nonlinearity, Integrated deformation energy

\section{Introduction}

Over the past three decades, topology optimization has gained wide-spread popularity in academic research and industrial applications, and proven its capabilities in advanced multidisciplinary fields and complicated solutions for practical engineering problem. Recent achievements are summarized in the four review papers [1-4]. Generally, topology optimization is an efficient way to seek the optimized material distribution in a given design space with the goal of improving structural performances along with one or more constraints, such as displacements [5, 6], maximum stress $[7,8]$ or bearing loads $[9,10]$, etc.

\footnotetext{
${ }^{*}$ Corresponding author

Email addresses: liyu_npu@outlook.com (Yu Li), jh.zhu@nwpu.edu.cn (Jihong Zhu)
} 
In practical structural design processes, engineers often require stiffer design results with limited material consumption, i.e. the standard compliance-based topology optimization design formulation. With an objective function of minimizing the global structural compliance, the optimized design result provides a stiff conceptual material layout, which gives guidance for further detail designs. For example, Krog et al. [11] applied a conventional compliance-based topology optimization method to the design of aircraft wing box ribs. Internal load paths in the wing box were automatically updated using a standard compliance-based topology optimization formulation. Recently, Aage et al. [12] demonstrated a topology optimization design of the internal structure of a full-scale airplane wing. The design problem considered multiple aerodynamic loading scenarios with a volume constraint and appropriate boundary conditions. Supported by a supercomputer, giga-voxel resolution produced impressive structural details covering multiple length scales, such as curved spars and diagonally placed ribs.

Moreover, researchers have considered control of structural deformation patterns. For example, Maute and Allen [13] studied wing stiffener layout using topology optimization under aerodynamic loads. An aerodynamically compatible shape was chosen as the objective shape and was preserved by constraining the nodal displacements of the wing tips. Zuo and Xie [14] used a minmax global or sub-domain displacement control to satisfy functional requirements. Therein, a set of nodal displacements was chosen as the constrained set to control position variations.

In many industrial cases such as the design, manufacturing, and assembling of aircraft structures, engineers face challenges in suppressing local warping deformation in order to maintain precise shapes for geometry features or functional surfaces. An example could be an antenna that needs to remain undistorted during deformations of the airplane structure or windows or cabin doors that need to be shielded from outer loads, c.f. Figure 1a. Traditional constraints on nodal displacements or global objective measures cannot describe local deformation behavior properly. Therefore, in the earlier work of Zhu et al. [15], such local deformation issues were characterized as shape preserving problems and solved by the proposed shape preserving design approach. Li et al. [16] further investigated specific directional local deformation control. Lately, Castro et al. [17] extended the shape preserving approach to dynamic problems.
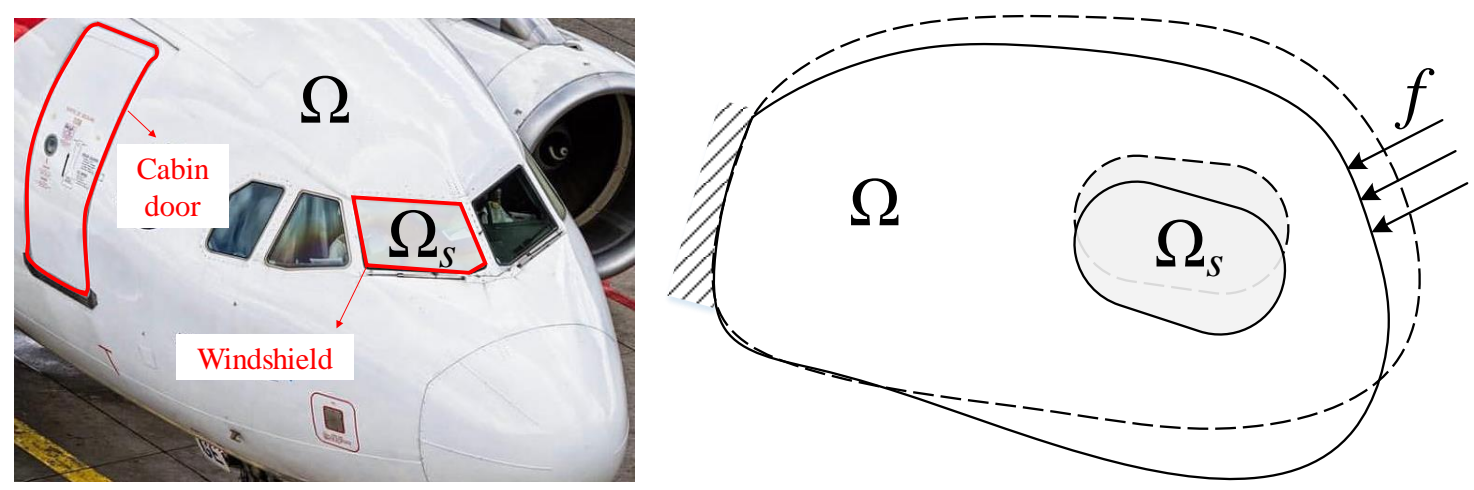

Figure 1: a) Illustration of need for shape preserving domains in the form of window and door regions in airplanes; $b$ ) Definition of design domain and shape preserving domains

Until now, shape preserving design has only been utilized with the assumption of linear elastic 
deformations. For some design applications in engineering fields such as soft or slender structure and compliant mechanism, the assumption of small deformations is not valid. And importantly, compared to small displacements conditions, more serious problems of local warping deformation will occur in structures undergoing large deformations. Therefore, it is necessary to further extend the current shape preserving design approach to problems with geometrical nonlinearity. Challenges regarding appropriate choice of objective functions and nonlinear warping constraints make the extension non-trivial compared to the linear case.

In numerical implementation of fictitious domain topology optimization methods with structures undergoing large displacements, a challenging problem has been excessive distortions in low stiffness elements resulting in non-convergence of the nonlinear finite element solver. Several strategies have been developed to circumvent the numerical instability. Buhl et al. [18] proposed to relax the convergence criterion for the equilibrium iterations by ignoring nonstructural nodes surrounded by low density elements. Bruns and Tortorelli [19] developed a method to remove and reintroduce low density elements during the optimization process. Yoon and Kim [20] introduced a connectivity parametrization, where all elements were connected by zero-length elastic springs. Klarbring [21] employed hyperelastic material models with a more physical behavior in compression. Wang et al. [22] proposed an energy interpolation scheme, where the strain energy densities in solid and void regions were interpolated using the strain energy densities for large and small deformation theories, respectively. Luo et al. [23] proposed to resolve the numeral difficulties by adding a special hyperelastic material to the design domain.

In this work, the energy interpolation scheme [22] is applied to alleviate numerical instabilities considering its generality and robustness for general topology optimization problems. Based on a hyperelastic strain energy function, which describes material behavior under large deformation and is a function of the Green-Lagrange strain [19, 21, 22], an improved warpage formulation weighted by local stiffness is introduced to calculate the geometrical distortion in shape preserving regions. To guarantee a stable solution and practical engineering results, the structural complementary elastic work is assigned as the objective function. The shape preserving design formulation is further established with a proper constraint value based on the integrated deformation energy over load increments. Isolation phenomena are considered and the influence of varying local stiffness is investigated as well. The effectiveness of the shape preserving design approach is validated by several numerical examples.

The remainder of this paper is organized as follows. Section 2 presents the definition of the shape preserving design problem considering geometrical nonlinearity. Section 3 describes density and energy interpolation schemes along with the associated sensitivity analyses. Section 4 presents numerical examples. Conclusions are drawn in Section 5.

\section{Shape preserving design problem considering geometrical nonlinearity}

Shape preserving design with structural topology optimization concerns suppressing warping deformation in shape preserving sub domains $\Omega_{s}$ while maximizing overall stiffness, c.f. Figure $1 b$ for an illustration. The optimization formulation is modified compared to the previously developed approach for linear analysis [15]. This section first sets up the optimization problem and then discusses and motivates its individual parts in subsequent text and subsections. 
The optimization problem is written as

$$
\begin{array}{cl}
\underset{\rho}{\min } & W_{\Omega}^{\mathrm{c}}(\boldsymbol{\rho}), \\
\text { s.t. } & \boldsymbol{r}(\boldsymbol{\rho}, \boldsymbol{u})=\mathbf{0}, \\
& V(\boldsymbol{\rho}) \leq V^{*}, \\
& \frac{\hat{\Phi}_{\Omega_{s}}(\boldsymbol{\rho}) / V_{\Omega_{s}}}{\Phi_{\Omega}(\boldsymbol{\rho}) / V^{*}} \leq \varepsilon_{\mathrm{sp}}, \\
& W_{\Omega}^{\mathrm{cA}}(\boldsymbol{\rho}) \leq W_{\Omega}^{\mathrm{cA}}, \\
& 0 \leq \rho_{e} \leq 1 \quad e=1, \ldots, n_{e} .
\end{array}
$$

Here, $\boldsymbol{\rho}$ is the design variable vector containing $n_{e}$ element-based design variables $\rho_{e}$, describing the material distribution in the design domain $\Omega$. $W_{\Omega}^{\mathrm{c}}$ is the complementary work of external forces in the total domain $\Omega$. $\Phi_{\Omega}$ is the deformation energy integrated over the load history, which is identical to the work of external forces, $W_{\Omega}$, (see e.g. [24]). $W_{\Omega}, W_{\Omega}^{\mathrm{c}}$ and $\Phi_{\Omega}$ are identical in the linear case. In the case of geometrical nonlinearity, they are normally different and share the relationship $W_{\Omega}^{\mathrm{c}}+W_{\Omega}=W_{\Omega}^{\mathrm{c}}+\Phi_{\Omega}=C_{\Omega}$, where $C_{\Omega}$ is the global end-compliance to be defined later. $\boldsymbol{r}$ is the residual in obtaining the nonlinear structural equilibrium with $\boldsymbol{u}$ denoting the displacement vector. $\boldsymbol{u}$ does not enter as optimization variable since it is calculated in an external finite element step using a nested approach [25]. $V$ and $V^{*}$ are the material volumes of the design domain and its prescribed upper bound, respectively. $V_{\Omega_{s}}$ is the volume of the shape preserving domain. The warping of the shape preserving domain $\hat{\Phi}_{\Omega_{s}}$ is measured by the normalized deformation energy integrated over the loading history. $\varepsilon_{\mathrm{sp}}$ is the corresponding shape preserving constraint value. $W_{\Omega}^{\mathrm{cA}}$ and $W_{\Omega}^{\mathrm{cA} *}$ are the structural complementary elastic work analyzed in an additional load case and its upper bound, respectively, introduced to avoid isolation of the shape preserving domain in specific cases. Detailed discussions and calculations of all the quantities used here are presented below and in Secs. 2.1 and 2.2.

Using a finite element discretization, the structural equilibrium, Eq. (1b), is written as

$$
r=f^{\text {ext }}-f^{\text {int }}(\rho, u)=0,
$$

where $f^{\text {ext }}$ is the external nodal load vector and $f^{\text {int }}=\sum_{e} f_{e}^{\text {int }}$ the internal nodal load vector. In this paper, the external forces are assumed to be design- and displacement- independent. The elemental internal force vector is expressed by

$$
\boldsymbol{f}_{e}^{\mathrm{int}}=\frac{\partial \int_{\Omega_{e}} \phi(\boldsymbol{u}) \mathrm{d} \Omega_{e}}{\partial \boldsymbol{u}_{e}},
$$

where $\phi(\boldsymbol{u})$ is the hyperelastic strain energy density function that describes the material behavior and $\boldsymbol{u}_{e}$ is the nodal displacement vector of element $e$. To emphasize generality, we leave for now the specific choice of strain energy density function $\phi(\boldsymbol{u})$ open and hence only decide on a specific form in the example section.

The nonlinear system, Eq. (2), is solved using the Newton-Raphson method with the incremental equation given as

$$
\boldsymbol{K}^{\tan } \Delta \boldsymbol{u}=\boldsymbol{r},
$$


where $\boldsymbol{K}^{\mathrm{tan}}$ is the tangent stiffness matrix defined as

$$
\boldsymbol{K}^{\tan }=-\frac{\partial \boldsymbol{r}}{\partial \boldsymbol{u}}
$$

The nodal displacement vector is updated by $\boldsymbol{u}=\boldsymbol{u}+\Delta \boldsymbol{u}$. Detailed computations of the tangent matrix and the nodal force vectors can be found in Zienkiewicz et al. [26].

\subsection{Objective function}

For topology optimization with geometrical nonlinearity, there are several possible definitions of the objective function. Other works have considered both end-compliance and end-stiffness objectives, however, these measures may overlook excessive deformations during loading (c.f. example discussed in Section 4.4 and associated load-displacement plot in Figure 23). Hence, the structurally most reasonable objective is the minimization of the structural complementary elastic work $[18,24]$ of external forces $f^{\text {ext }}$, which can be calculated using the trapezoidal rule as

$$
W_{\Omega}^{\mathrm{c}}=\int_{\mathbf{0}}^{f^{\mathrm{ext}}}(\boldsymbol{u}(\boldsymbol{f}))^{\mathrm{T}} \mathrm{d} \boldsymbol{f} \approx \Delta \boldsymbol{f}^{\mathrm{T}}\left(\sum_{i=1}^{n-1} \boldsymbol{u}_{i}+\frac{1}{2} \boldsymbol{u}_{n}\right),
$$

where $n$ is the number of load increments, $\Delta \boldsymbol{f}$ is the increment size determined by $\Delta \boldsymbol{f}=\boldsymbol{f}^{\mathrm{ext}} / n$. $\boldsymbol{u}_{n}$ is the final displacement vector from the incremental loading process and $\boldsymbol{u}_{i}$ is the corresponding displacement vector at the $i$ th load increment. Using this goal, the optimization will result in structurally sound results within the incremental history up to the design load. The alternative definitions of objective function such as end-compliance [18, 22, 23] or alternatively, endstiffness $[27,28]$, are much simpler and more direct in calculation but may produce less sensible designs as elaborated below.

The end-compliance function can be expressed as

$$
C_{\Omega}=\left(f^{\text {ext }}\right)^{\mathrm{T}} \boldsymbol{u}_{n}
$$

which is only calculated in the final equilibrium, i.e. in the $n$th incremental load step. Therefore, it can be considered as a particular form of the complementary elastic work with only one load increment. However, this definition, as well as the end-stiffness may result in structures that are only optimized for the prescribed load but have non-proportional and excessive displacements for smaller loads, c.f. [18]. Thus here we focus on the minimization of the complementary elastic work. Nevertheless, we also include a numerical example minimizing the structural endcompliance for completeness and to demonstrate its deficiencies in Sec. 4.4.

To complete this subsection, we also define the integrated deformation energy as

$$
\Phi_{\Omega}=\int_{\Omega} \int_{0}^{\boldsymbol{u}_{n}}\left(\frac{\partial \phi(\boldsymbol{u})}{\partial \boldsymbol{u}}\right)^{\mathrm{T}} \mathrm{d} \boldsymbol{u} \mathrm{d} \Omega=\int_{\mathbf{0}}^{\boldsymbol{u}_{n}}\left(f^{\mathrm{int}}(\boldsymbol{u})\right)^{\mathrm{T}} \mathrm{d} \boldsymbol{u},
$$

and the work of external forces as [24]

$$
W_{\Omega}=\int_{0}^{\boldsymbol{u}_{n}}\left(\boldsymbol{f}^{\mathrm{ext}}(\boldsymbol{u})\right)^{\mathrm{T}} \mathrm{d} \boldsymbol{u},
$$


where $\boldsymbol{f}^{\mathrm{ext}}(\boldsymbol{u})$ means the corresponding incremental external force during load increments. Note that, the integrated deformation energy is a self-defined energy function, which is based on the nonlinear energy density $\phi(\boldsymbol{u})$. It is in this study proposed to calculate the stored energy during the whole loading process. Based on Eqs. (2), (8) and (9), one can conclude that the integrated deformation energy over the whole domain, $\Phi_{\Omega}$, is identical to the work of external forces, $W_{\Omega}$. Finally, we repeat the relation between above defined measures as $W_{\Omega}^{\mathrm{c}}+W_{\Omega}=W_{\Omega}^{\mathrm{c}}+\Phi_{\Omega}=C_{\Omega}$.

\subsection{Shape preserving constraint function}

In order to acquire a useful measure of the warping deformation under geometrical nonlinearity, we here use the hyperelastic strain energy $\Phi$. As shown in Figure 2, the strain energy of a $0.01 \mathrm{~m} \times 0.01 \mathrm{~m}$ plane element with a thickness of $0.001 \mathrm{~m}$ after a rotation of 10 degrees is calculated to be $0 \mathrm{~kJ}$ by a nonlinear FEA method, while linear FEA, $\Phi_{L}$, produces a non-zero result. Thus, the constraint function based on a linear strain energy measure as used in previous linear cases $[15,16]$, would restrain also rigid body rotation and hence may not serve as a proper constraint for nonlinear shape preserving purposes.

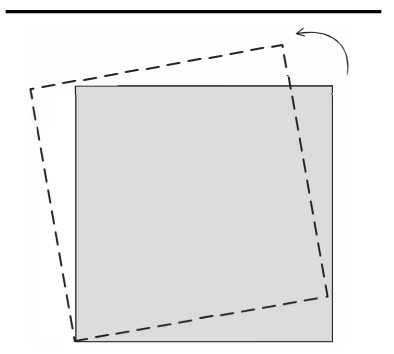

Young's modulus: $210 \mathrm{GPa}$

Poisson's ratio: 0.3

\begin{tabular}{cc}
\hline$\Phi_{L}$ & $\Phi$ \\
\hline $6.92 \mathrm{~kJ}$ & $0 \mathrm{~kJ}$ \\
\hline
\end{tabular}

Figure 2: FEA results for rigid body rotation

Instead we propose a refined criterion for a normalized warpage function $\hat{\Phi}_{\Omega_{s}}$ based on the integrated deformation energy over the shape preserving domain $\Phi_{\Omega_{s}}$. This can be written as

$$
\hat{\Phi}_{\Omega_{s}}=\frac{\Phi_{\Omega_{s}}}{E_{\Omega_{s}}} E_{1}
$$

where $E_{\Omega_{s}}$ is the Young's modulus in the shape preserving domain and $E_{1}$ is the Young's modulus of the base material in the general design domain. Here, $E_{1}$ serves as a normalization factor which makes the warpage measure directly comparable to the overall integrated deformation energy and hence makes the choice of constraint value simpler. Also, the normalized criterion value is independent of the local stiffness and hence yields a stiffness independent measure of the geometrical distortion. This effect will be discussed further and demonstrated in Sec. 4.1. 
Similar to the integrated deformation energy over the whole domain (8), the integrated deformation energy in the shape preserving domain is defined as

$$
\Phi_{\Omega_{s}}=\int_{\Omega_{s}} \int_{0}^{\boldsymbol{u}_{n}}\left(\frac{\partial \phi(\boldsymbol{u})}{\partial \boldsymbol{u}}\right)^{\mathrm{T}} \mathrm{d} \boldsymbol{u} \mathrm{d} \Omega_{s}=\int_{0}^{\boldsymbol{u}_{n}}\left(f_{\Omega_{s}}^{\mathrm{int}}(\boldsymbol{u})\right)^{\mathrm{T}} \mathrm{d} \boldsymbol{u},
$$

where $f_{\Omega_{s}}^{\text {int }}$ is the internal nodal force vector in $\Omega_{s}$. It can be seen that the integrated deformation energy is calculated with the shape preserving domain's internal force vector, which depends on the loading history. Further details of this computation and the associated sensitivity analysis can be found in Appendix A.

As shown in Eq. (1d), the warpage energy constraint function is implemented with a relative constraint value $\varepsilon_{\mathrm{sp}}$ between the average warping energy $\hat{\Phi}_{\Omega_{s}} / V_{\Omega_{s}}$ in the shape preserving domain and the global average warping energy $\Phi_{\Omega} / V^{*}$. Using the averaged energies makes the constraint independent on volume fractions. With a properly chosen constraint value $\varepsilon_{\mathrm{sp}}$ in Eq. (1d), the average level of warping energy in $\Omega_{s}$ is suppressed to a proportion of the global average warping energy. As a desired result, the deformation of the considered shape preserving subdomain is constrained to near rigid body movements.

As described so far, the calculation of the integrated deformation energy in the shape preserving domain is based on the accurate deformation pattern of the shape preserving domain as shown in Figure 3 (two leftmost subfigures). Alternatively, to deal with the situation where the shape preserving domain is only connected to the main structure in discrete points, the integrated deformation energy calculation in the shape preserving domain may be based on much coarser measurements using a few key points as shown in Figure 3 (two rightmost subfigures). With this alternative representation the shape preserving domain approach is changed into a multi-point one and the multi-point deformation energy is calculated based on the corresponding internal forces of the coarse elements with the same material properties as the shape preserving domain. Apart from allowing increased flexibility in the control of the shape measure, the alternative approach also caters for the special situation where the shape preserving domain is void (or $E_{\Omega_{s}}=10^{-9}$ ). In this case, the key points can be selected as some boundary nodes describing only the perimeter of the shape preserving domain. Relevant multi-point design results are presented in Sec. 4.3.

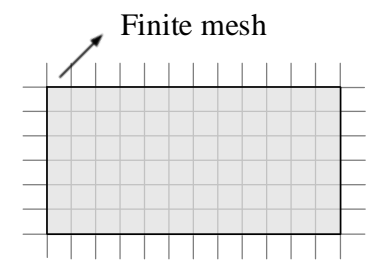

Shape preserving domain

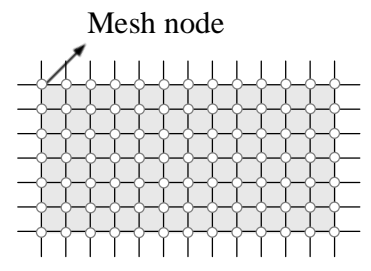

Full measurement

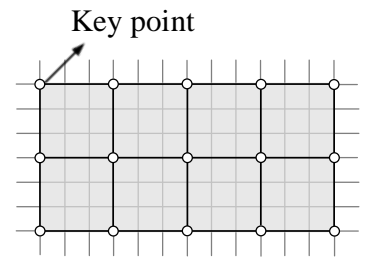

Coarse measurement 1

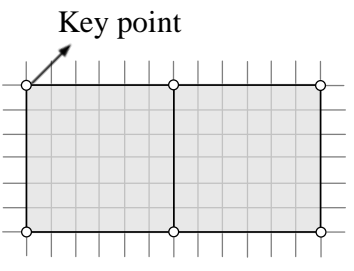

Coarse measurement 2

Figure 3: Full and coarse measurements in shape preserving domain

\subsection{Prevention of isolated shape preserving domains}

The constraint function with an additional load condition, Eq. (1e), is added to the formulation to avoid the shape preserving domain to be isolated. The problem to be avoided is illustrated in 
Figure 4(a), where a cantilever beam is subjected to evenly distributed horizontal stretching force of $f=10 \mathrm{kN}$. The integrated deformation energy in the shape preserving domain is constrained to eliminate local warping deformation. The non-surprising optimized design is shown in Figure 4(b). Naturally, the shape preserving domain is isolated from the supporting structure and thus totally undeformed. Such a solution, where the integrated deformation energy in the shape preserving domain is zero, is mathematically feasible, but has no engineering value.

Therefore, an additional load case is introduced to circumvent disconnected shape preserving domains. As shown in Figure 5(a), a small downward concentrated force (or alternatively a gravitational load) of $f_{\text {Add }}=0.1 \mathrm{kN}$, i.e. $1 \%$ of the external load, is added to the centroid of the shape preserving domain. With Eq. (1e), a constraint on the complementary work produced by this additional load case is included in the optimization problem, where the upper bound value $W_{\Omega}^{\mathrm{cA}}{ }^{*}$ is set to 0.1 (100 times the initial one). Since the magnitude of this additional load case is relatively small compared to the major load scenario and it mainly serves to ensure connectiveness of the local region, the analysis of this additional load case is linear. The corresponding optimized design is shown in Figure 5(b). The additional constraint results in an optimized design where the shape preserving domain now is connected to the main structure.

Remark, however, that the deformation pattern of this stretching force example is quite simple, and hence provokes the unwanted island. For other design problems with more complex geometries or load conditions we did not observe the island issue and hence the additional constraint function is inactive. This holds for all other examples considered here. In practice, the extra constraint may only be needed under very special circumstances.

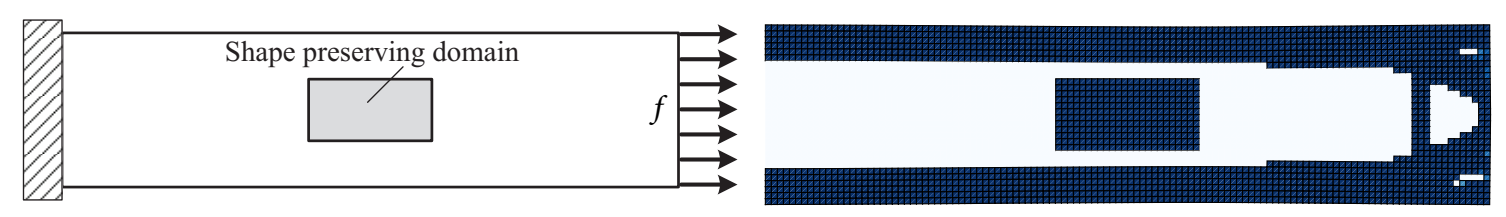

(a) A cantilever beam under horizontal extension (b) Shape preserving design result with isolated soluloads. tion.

Figure 4: The isolation phenomenon in shape preserving design

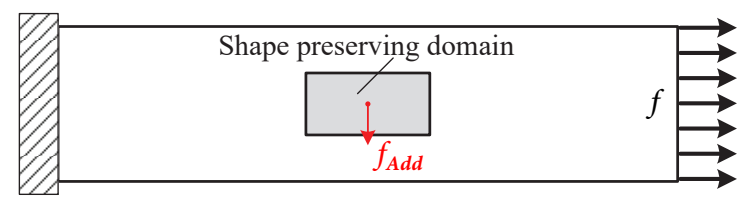

(a) Additional load on the shape preserving domain.

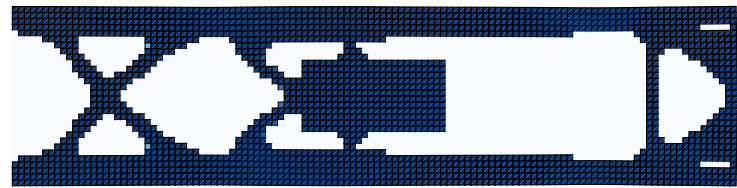

(b) Shape preserving design result without isolated solution.

Figure 5: Shape preserving design with added avoidance of isolation constraint 


\section{Interpolation methods and sensitivity analysis}

\subsection{Density interpolation and projection filtering}

The following modified SIMP interpolation scheme [29] is used to relate the physical density of element $e, \bar{\rho}_{e}$, and the Young's modulus. This is expressed as

$$
E_{e}=E\left(\bar{\rho}_{e}\right)=E_{0}+\bar{\rho}_{e}^{p}\left(E_{1}-E_{0}\right) \quad 0 \leq \bar{\rho}_{e} \leq 1,
$$

where $E_{1}$ is the Young's modulus of the solid material in the design domain, $E_{0}$ is the Young's modulus of void, which is non-zero to avoid the singularity of the tangent stiffness matrix (here $\left.E_{0}=10^{-9} E_{1}\right) \cdot p$ is the penalty factor.

To promote discrete final designs, the physical density is represented by a smoothed Heaviside projection function $[22,30]$ based on the hyperbolic tangent function, stated as

$$
\bar{\rho}_{e}=\frac{\tanh (\beta \eta)+\tanh \left(\beta\left(\tilde{\rho}_{e}-\eta\right)\right)}{\tanh (\beta \eta)+\tanh (\beta(1-\eta))}
$$

where $\tilde{\rho}_{e}$ is the filtered density of element $e . \beta$ controls the sharpness of the projection and $\eta$ is the threshold value between 0 to 1 . When $\beta \rightarrow \infty$, designs are forced towards pure $0-1$ solutions. The filtered density is calculated as

$$
\tilde{\rho}_{e}=\frac{\sum_{j \in N_{e}} w_{e}\left(\boldsymbol{x}_{j}\right) v_{j} \rho_{j}}{\sum_{j \in N_{e}} w_{e}\left(\boldsymbol{x}_{j}\right) v_{j}},
$$

where $\boldsymbol{x}_{j}$ are the center coordinates of element $j . v_{j}$ and $\rho_{j}$ are the volume and design variable value of element $j$, respectively. $N_{e}$ is the neighborhood of element $e$ within a certain filter radius specified by $N_{e}=\left\{j \mid\left\|\boldsymbol{x}_{j}-\boldsymbol{x}_{e}\right\| \leq r\right\}$, and $w_{e}\left(\boldsymbol{x}_{j}\right)=r-\left\|\boldsymbol{x}_{j}-\boldsymbol{x}_{e}\right\|$.

In all examples in this paper, the radius $r$ in the density filter is set to 3.5 times the average element size, and the threshold value $\eta$ is set to 0.5. A continuation scheme proposed in [22] is applied to the penalization parameter $p$ to avoid instabilities associated with the original grey starting guess (starting with a uniform porous starting guess and high penalization factor results in an extremely soft structure for which finite element convergence cannot be achieved). $p$ is hence raised in steps of 0.05 from 1.0 to 3.0, every two iterations when $p<2.0$ and every five iterations when $p \geq 2.0$. To obtain a better $0-1$ convergence, a continuation scheme is also employed to increase $\beta$ in Eq. (13) after $p=3.0$. $\beta$ is updated every 10 iterations from 4 to 64 using $\beta=2 \times \beta$. For clarity, only elements with a physical density equal to or bigger than 0.5 are presented in the visualizations of the design results, although we remark that due to the projection approach there remain very few elements with intermediate densities in the optimized results.

\subsection{Energy interpolation scheme}

To avoid numerical instabilities induced by excessive deformations in low stiffness regions during the topology optimization process, the energy interpolation scheme proposed by Wang et al. [22] is implemented. The strain energy density in a solid or intermediate density element corresponds to the nonlinear one, whereas the strain energy density in a void element simply 
corresponds to the linear one considering small deformations. This energy interpolation form for element $e$ is expressed as

$$
\phi_{e}\left(\boldsymbol{u}_{e}\right)=\left[\phi_{H}\left(\gamma_{e} \boldsymbol{u}_{e}\right)-\phi_{L}\left(\gamma_{e} \boldsymbol{u}_{e}\right)+\phi_{L}\left(\boldsymbol{u}_{e}\right)\right] E_{e},
$$

where $\boldsymbol{u}_{e}$ is the elemental nodal displacement vector. $\phi_{H}(\cdot)$ refers to the hyperelastic strain energy density and $\phi_{L}(\cdot)$ is the linear one under small deformation, both with unit Young's modulus. This interpolation scheme assumes that the Young's modulus is separable from the energy function. Based on Eqs. (3), (12) and (15), the relationship between the elemental internal force vector and physical density variable can be simply expressed as

$$
f_{e}^{\text {int }}=E\left(\bar{\rho}_{e}\right) \boldsymbol{f}_{e 0}^{\text {int }},
$$

where $f_{e 0}^{\text {int }}$ is the internal force result of the unit Young's modulus of element $e$.

The interpolation factor $\gamma_{e}$ is defined as a threshold, again using a smoothed Heaviside projection function to ensure a smooth and differentiable transition

$$
\gamma_{e}=\frac{\tanh \left(\beta_{1} \rho_{0}\right)+\tanh \left(\beta_{1}\left(\bar{\rho}_{e}^{p}-\rho_{0}\right)\right)}{\tanh \left(\beta_{1} \rho_{0}\right)+\tanh \left(\beta_{1}\left(1-\rho_{0}\right)\right)},
$$

where $\gamma_{e}$ is 1 for elements with $\bar{\rho}_{e}^{p}>\rho_{0}$ and 0 for $\bar{\rho}_{e}^{p}<\rho_{0}$. The control parameters in Eq. (17) are fixed to be $\rho_{0}=0.01$ and $\beta_{1}=500$ for all examples as suggested in [22].

Note that above energy interpolation scheme is also used in the structural analysis of the shape preserving domain, i.e. for very low stiffness of the shape preserving domain, it is modelled using linear strain energy density $\phi_{L}$. However, in order to model both rigid motion and rotation, the corresponding strain energy in the shape preserving constraint function is still calculated based on the nonlinear one, i.e. the original $\phi\left(\boldsymbol{u}_{e}\right)$ without the interpolation of Eq. (17).

\subsection{Sensitivity analysis}

The optimization problem in this paper is a typical nonlinear programming problem solved using a gradient based method, where the search for the optimized design takes place along a single path whose trajectory is determined by the gradients of the objective and constraint functions. The efficient adjoint method is utilized for computing the gradients of all structural functions $\mathcal{F}$ with respect to the elemental physical density $\bar{\rho}_{e}$. All the derivations are summation results related to load increments, i.e.

$$
\frac{\mathrm{d} \mathcal{F}}{\mathrm{d} \bar{\rho}_{e}}=\frac{\partial \mathcal{F}(\boldsymbol{u}, \overline{\boldsymbol{\rho}})}{\partial \bar{\rho}_{e}}+\sum_{i=1}^{n} \lambda_{i}^{\mathrm{T}} \frac{\partial \boldsymbol{r}_{i}}{\partial \bar{\rho}_{e}},
$$

where $\lambda_{i}$ and $\boldsymbol{r}_{i}$ are the adjoint variable vector and the nodal residual force vector in the $i$ th incremental load step, respectively. $\lambda_{i}$ is obtained by solving the linear system solution as

$$
\boldsymbol{K}_{i}^{\tan } \boldsymbol{\lambda}_{i}=\left(\frac{\partial \mathcal{F}\left(\boldsymbol{u}_{i}, \overline{\boldsymbol{\rho}}\right)}{\partial \boldsymbol{u}_{i}}\right)^{\mathrm{T}},
$$


where $\boldsymbol{K}_{i}^{\mathrm{tan}}$ is the tangent stiffness at the $i$ th converged solution with the corresponding displacement vector $\boldsymbol{u}_{i}$. Considering the energy interpolation scheme, $\partial \boldsymbol{r}_{i} / \partial \bar{\rho}_{e}$ is further calculated as

$$
\frac{\partial \boldsymbol{r}_{i}}{\partial \bar{\rho}_{e}}=-\left(\frac{\partial \boldsymbol{f}_{i}^{\text {int }}}{\partial \bar{\rho}_{e}}+\frac{\partial \boldsymbol{f}_{i}^{\text {int }}}{\partial \gamma_{e}} \frac{\partial \gamma_{e}}{\partial \bar{\rho}_{e}}\right)
$$

where $f_{i}^{\text {int }}$ is the internal nodal force vector in the $i$ th incremental load step.

The final sensitivity of $\mathcal{F}$ with respect to the design variable $\rho_{e}$ is calculated following the chain rule [22], i.e.

$$
\frac{\mathrm{d} \mathcal{F}}{\mathrm{d} \rho_{e}}=\sum_{j \in N_{e}} \frac{\mathrm{d} \mathcal{F}_{j}}{\mathrm{~d} \bar{\rho}_{j}} \frac{\mathrm{d} \bar{\rho}_{j}}{\mathrm{~d} \tilde{\rho}_{j}} \frac{\mathrm{d} \tilde{\rho}_{j}}{\mathrm{~d} \rho_{e}},
$$

where $\mathcal{F}_{j}$ is the elemental design functions of element $j$.

Detailed derivations of this sensitivity analysis for the objective function of the complementary elastic work as well as the end-compliance can be found in [18]. The sensitivity analysis of the shape preserving constraint function with the integrated deformation energy is presented in Appendix A. Based on the sensitivity analyses, the structure is iteratively updated using the Method of Moving Asymptotes (MMA) [31].

\section{Numerical results of shape preserving design}

In this section, shape preserving design with geometrical nonlinearity is tested on two classical 2D problems. Multi-point shape preserving designs with void regions are studied as a special case. Formulations and derivations in the previous sections assumed an arbitrary hyperelastic material model. In the following examples, the classical Saint Venant-Kirchhoff strain energy density function is used to describe material behavior of the base material. This is a valid model when considering large displacements but small strains as verified for studies with similar parameters but without shape preserving domains [22]. Potentially, it is straight forward to substitute the simple Saint Venant-Kirchhoff model with other more advanced materials laws but this is not expected to change the present optimization results in any significant way as discussed also in [22]. In that paper it was shown that despite large deformations, strains stay small in load carrying solid regions and hence alternative material laws only influence low-density regions and hence algorithmic convergence and not structural performance.

Shape preserving domains are non-designable. The base material used in this section is Nylon with Young's modulus $E_{1}=3.0 \mathrm{GPa}$ and Poisson's ratio $v=0.4$. Numerical solutions are obtained using equally sized load increments being $10 \mathrm{kN}$ under the plane strain assumption. The optimization procedure is implemented in a MATLAB platform and typically requires 500 iterations to converge. The number of Newton iteration steps per system solve is mostly below 5 and CPU time per optimization iteration is around 40 seconds. Clearly, code optimization or porting to a low level computing language could improve computational time considerably.

The optimization loop is terminated when the maximum absolute difference between two consecutive designs (in terms of design variables) is less than $1 \%$. The external move limit for updating the design variables in MMA is set as 0.05 . The starting design guess is a uniform density distribution satisfying the volume constraint in the design domain. 


\subsection{Cantilever beam with a rectangular shape preserving domain}

As shown in Figure 6, the first example considered has a rectangular shape preserving domain $(0.2 \mathrm{~m} \times 0.1 \mathrm{~m})$ embedded in the middle of a cantilever beam $(1.0 \mathrm{~m} \times 0.25 \mathrm{~m})$ with a thickness of $0.1 \mathrm{~m}$. The beam is clamped at the left edge and subjected to an external load of $f=150 \mathrm{kN}$ downwards at the midpoint of the right edge. The material property of the shape preserving domain is $E_{\Omega_{s}}=0.1 \times E_{1}=0.3 \mathrm{GPa}, v_{\Omega_{s}}=v=0.4$. The total structure is discretized using a mesh of $120 \times 30$ 4-node plane elements.

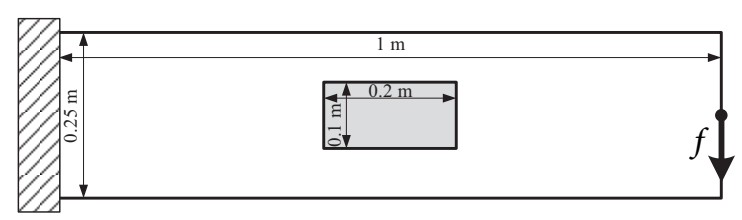

Figure 6: 2D cantilever beam example with a rectangular shape preserving domain

With a $50 \%$ material volume constraint, a standard design problem minimizing the global complementary elastic work without the shape preserving constraint is solved first. The corresponding (deformed) optimized design is shown in Figure 7. The resulting global complementary elastic work $W_{\Omega}^{\mathrm{c}}$ is $12.043 \mathrm{~kJ}$, while the normalized warping energy $\hat{\Phi}_{\Omega_{s}}$ in the shape preserving domain is calculated as $3.189 \mathrm{~kJ}$. The global integrated deformation energy $\Phi_{\Omega}$ is $11.785 \mathrm{~kJ}$ (corresponding to a relative warping measure of $156 \%$, c.f. Eq. (1d)). We observe that the resulting topology is slightly non-symmetric as expected, considering the rather large load applied. The deformed configuration of the shape preserving domain is compared to its original shape indicated by red lines. To further illustrate the effect of the shape preserving constraint, the normalized deformation energy distribution is also illustrated in the local deformation plot. This clearly shows that the sub-domain is undergoing large warping deformations that result in a distorted appearance.

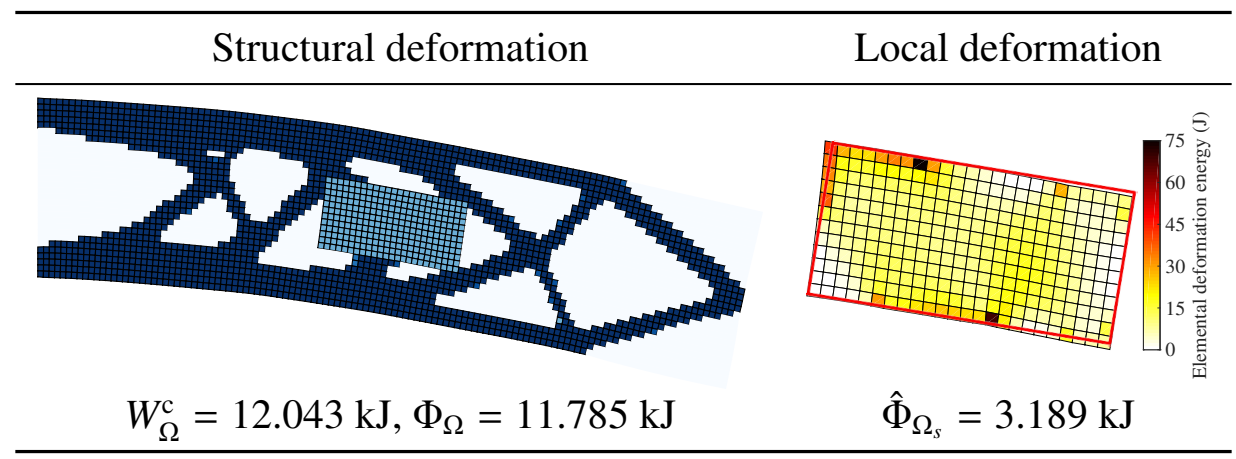

Figure 7: Standard design result for the cantilever beam with minimized $W_{\Omega}^{\mathrm{c}}$

To eliminate such undesired deformation, the shape preserving constraint is introduced with different upper bound values of $\varepsilon_{\mathrm{sp}}$ in Eq. (1d). Corresponding shape preserving design results under each constraint value are illustrated in Figure 8.

As shown in the local deformation result under the constraint value of $\varepsilon_{\mathrm{sp}}=25.0 \%$, the warping deformation in the rectangular block has mostly been suppressed. Nevertheless there is still 


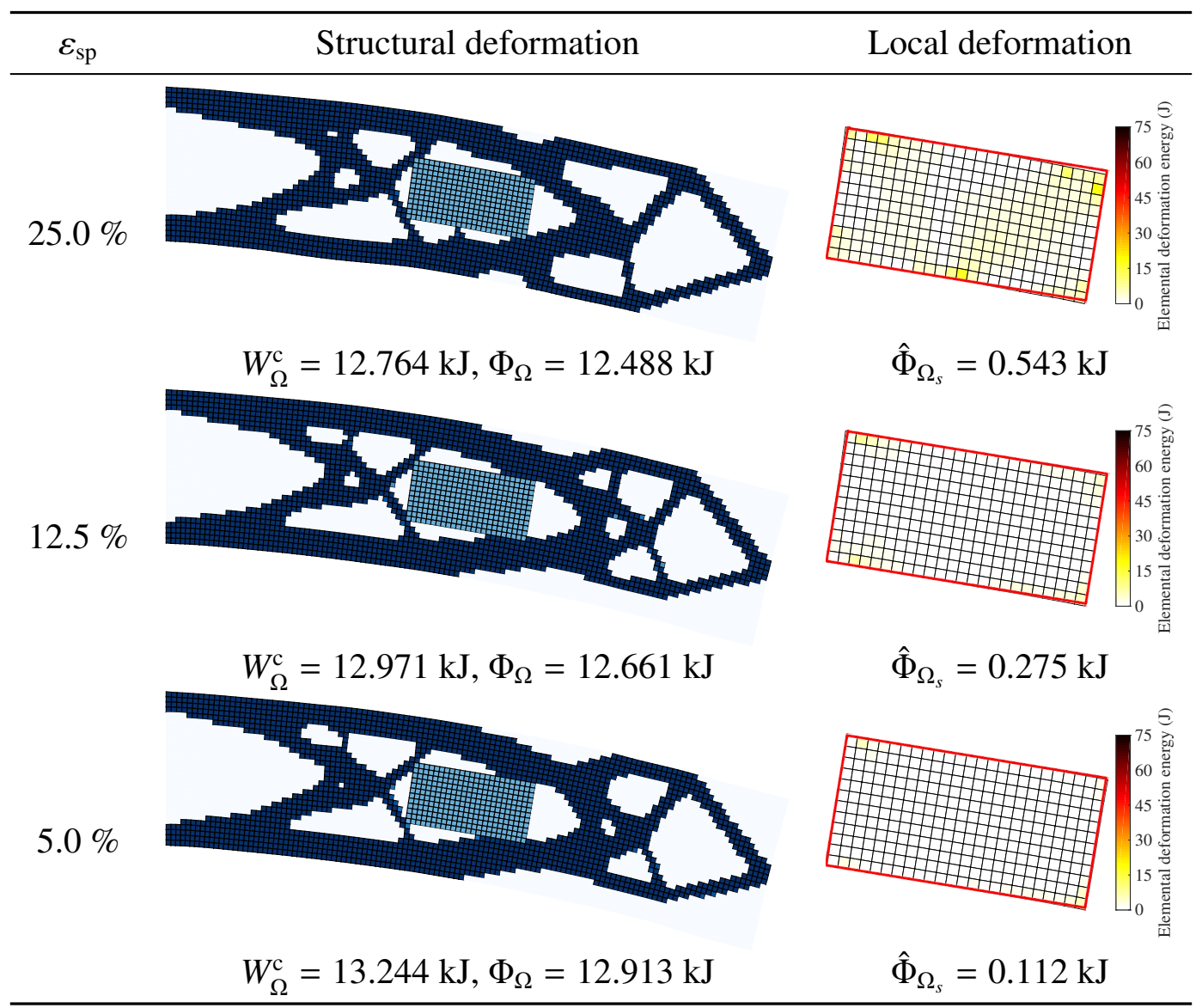

Figure 8: Shape preserving design results for the cantilever beam with minimized $W_{\Omega}^{\mathrm{c}}$ under different constraint values

some distortion in the top-right corner. To further improve the local performance, the constraint value is subsequently decreased to $12.5 \%$ and $5.0 \%$. For stronger enforcement of the energy constraint the design changes towards a shorter cantilever beam with an added thinner truss like extension structure. The load carrying path slickly circumvents the shape preserving domain, resulting in an almost perfect shape preserving effect. The corresponding optimized objective function and normalized warpage energy function are $13.244 \mathrm{~kJ}$ and $0.112 \mathrm{~kJ}$, respectively for the $5.0 \%$ case.

It is seen that the stronger the shape preserving constraint, the bigger the objective function. The enhancement of local shape preservation hence comes at the cost of decreased global stiffness. Therefore, a proper constraint value is important to determine the performances of shape preserving design results. After numerous tests, we suggest that an appropriate constraint value could be chosen between $50.0 \%$ and $5.0 \%$ depending on design case.

Figure 9 shows the evolution processes of the objective function and shape preserving constraint function for the three cases in Figure 8. In the $5.0 \%$ case, at the beginning of the optimization (e.g. before the $50^{\text {th }}$ iteration), the structure is rather weak and a too strict shape preserving constraint may jeopardize initial convergence. Thus, to ensure a stable optimization, a continuation method is also suggested to be applied on the shape preserving constraint value. For small constraint values (e.g. $15.0 \%$ to $5.0 \%$ ), the design process will firstly start from a larger one 


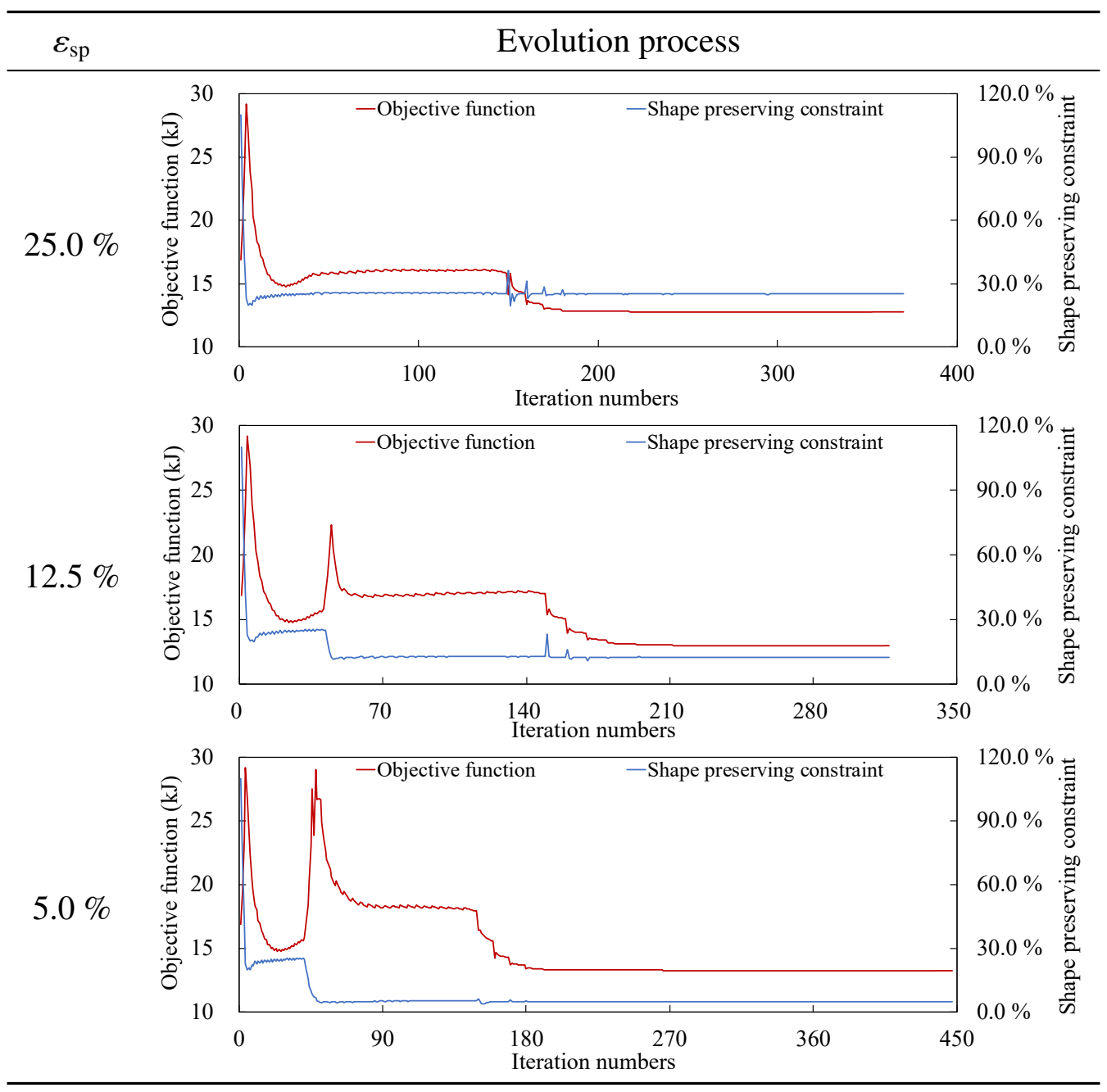

Figure 9: The evolution process of the objective function and constraint function of shape preserving design results in Figure 8

(e.g. $100.0 \%$ to $60.0 \%$ ) to obtain an intermediate result with sufficient structural stiffness before $p \leq 2$. Then the constraint value is gradually decreased in steps of -0.1 from the initial one to the final one, along with the increment of penalty $p$ until $p=3$.

As stated above and in Sec. 3.1, we apply a continuation approach on parameters $\varepsilon_{\mathrm{sp}}, p$ and $\beta$, to ensure best and most efficient convergence. These parameter updates are similar to those suggested in $[18,22]$ and the same settings are used for all the examples presented. To further indicate the robustness of the continuation approach applied on different parameters, both $25.0 \%$ and $5.0 \%$ cases in Figure 8 are resolved with a finer mesh and a starting guess of full solid material at the same time. The corresponding design results are shown in Figure 10. As expected, similar results are generated but with better objective functions.

Considering the plane strain assumption used in this paper, it is worth mentioning that the 2D hypothesis is an important point that must be considered in geometrically nonlinear design. As stated in [32], the optimized topologies and objective function values are different between the 
plane strain condition (PE) and plane stress condition (PS). Here, the $25.0 \%$ case and $5.0 \%$ case are resolved again with plane stress assumption. The design results are illustrated in Figure 11. As expected, the objective function value for the PS condition is higher than the one with PE. The compared results also show that the proposed shape preserving design method works well for both conditions with stable solutions.

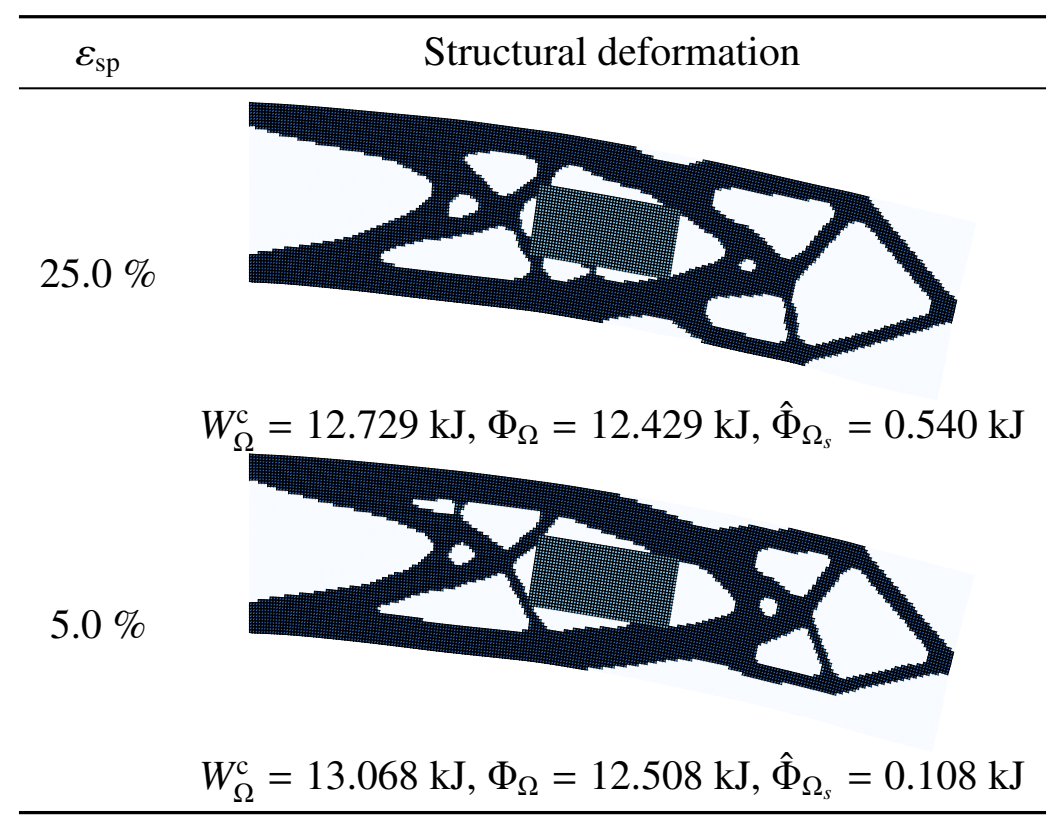

Figure 10: Shape preserving design results for the cantilever beam with a mesh of $240 \times 60$ and a starting guess of full solid material

\section{Influence of the stiffness of the shape preserving domain}

Next, the influence of different stiffnesses of the shape preserving domain is investigated based on the constraint value of $25.0 \%$. The stiffness of the shape preserving domain is represented by its Young's modulus $E_{\Omega_{s}}$ and can be defined with a linear relationship $\alpha$ to the structural stiffness $E_{1}$, i.e.

$$
E_{\Omega_{s}}=\alpha E_{1} .
$$

As shown in Figure 12, shape preserving designs under different stiffnesses of the shape preserving domain result in different optimized configurations, especially regarding the material layouts surrounding the shape preserving domains. In the figure, deformed solid regions are displayed in a lighter color corresponding to their Young's modulus. With a relatively high stiffness, the shape preserving domain is able to protect itself from distortion. Hence, in the design results for $\alpha=1$ and $\alpha=0.5$, structural stiffnesses are reinforced and the shape preserving block is only connected with some necessary branches. When the stiffness of the shape preserving domain is as weak as $1 \%$ of the design domain, the block is much more prone to warping deformation. Thus, the load carrying path around the shape preserving region is reinforced to prevent local deformation. As discussed in Sec 2.2, the shape preserving constraint function based on the normalized 


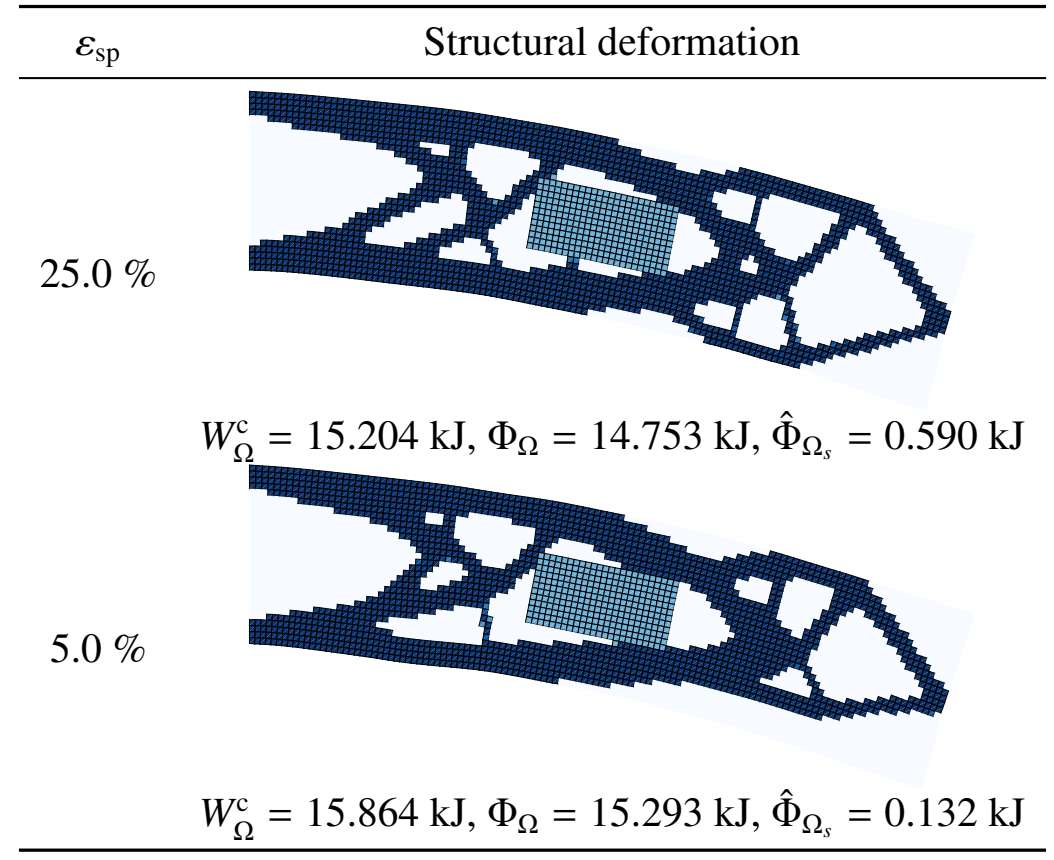

Figure 11: Shape preserving design results for the cantilever beam under plane stress assumption

deformation energy gives an equivalent warpage control for different domain stiffnesses. All of the geometrical warping deformation in shape preserving domains are equally avoided. The special case of void, i.e. $\alpha=10^{-9}$ will be presented in Sec. 4.3.

\subsection{L-shaped beam with a square shape preserving domain}

The second case study concerns an L-shaped beam with a shape preserving domain in the knee as shown in Figure 13. The beam with a thickness of $0.1 \mathrm{~m}$ is fixed at the upper edge and subjected to an external load of $f=100 \mathrm{kN}$ at the center of the right edge. Other geometrical dimensions are given in the figure. The shape preserving domain's material property is $E_{\Omega_{s}}=0.1 \times E_{1}=0.3$ $\mathrm{GPa}, v_{\Omega_{s}}=v=0.4$. The structure is discretized using a mesh with 80 by 804 -node plane elements subtracting a 48 by 48 void mesh of the upper right part of the beam.

The standard design result with minimized structural complementary work and a volume constraint value of $40 \%$ is shown in Figure 14. The final optimized $W_{\Omega}^{\mathrm{c}}$ becomes $2.962 \mathrm{~kJ}$, while the normalized warping energy $\hat{\Phi}_{\Omega_{s}}$ is $2.330 \mathrm{~kJ}$ with notable distortion in the shape preserving domain.

Thereafter, shape preserving designs under different constraint values are conducted to eliminate the local warping. Corresponding design results are shown in Figure 15. In the result of $\varepsilon_{\mathrm{sp}}=15.0 \%$, the shape preserving effect is well recovered, with the square moving in a rigid body fashion and its original shape preserved.

\subsection{Cantilever beam with a multi-point shape preserving domain}

Next, we revisit the cantilever example but with slightly changed geometry as shown in Figure 16. The shape preserving domain located in the middle square region is changed into void and a non-designable solid frame outlining the domain. The multi-point integrated deformation 


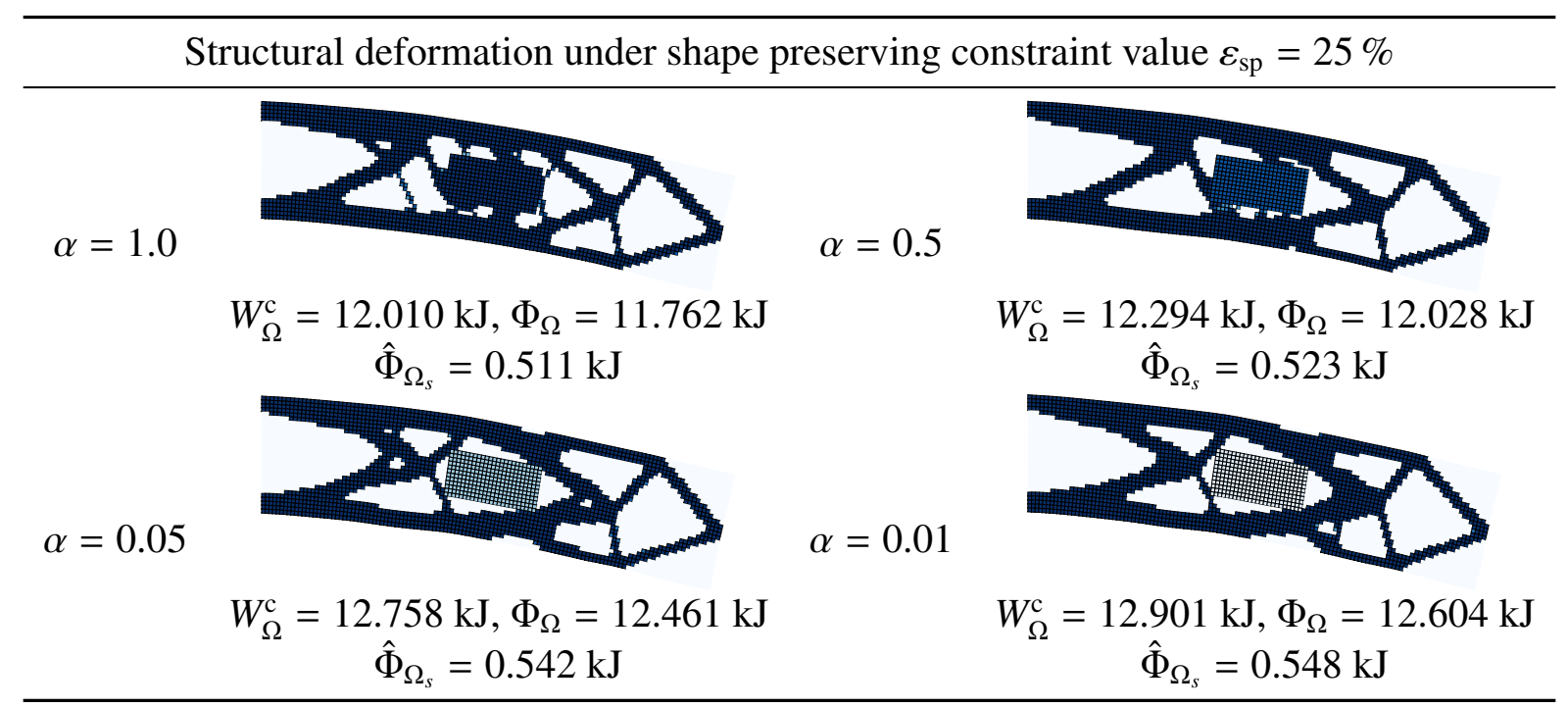

Figure 12: Shape preserving design results with varying local stiffnesses of the shape preserving domain.

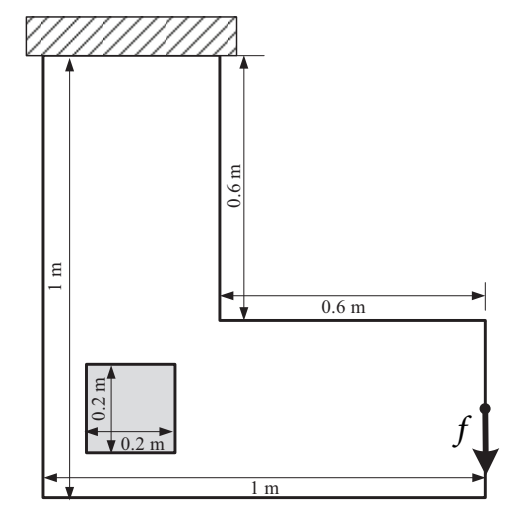

Figure 13: An L-shaped beam with a square shape preserving domain

energy $\Phi_{\Omega_{s}}$ is evaluated by two corresponding coarse elements and their six associated key points on the boundaries of the shape preserving domain. The (pseudo) stiffness of the void domain is $E_{\Omega_{s}}=E_{0}=10^{-9} E_{1}$, whereas the frame's material property is the same as the design structure, i.e. $E_{\text {Frame }}=E_{1}$.

First, the structure is optimized without the shape preserving constraint as presented in Figure 17. In the multi-point deformation results, deformed coarse elements are illustrated with black edges. The objective function $W_{\Omega}^{\mathrm{c}}$ is optimized to $12.507 \mathrm{~kJ}$ and the normalized warpage function $\hat{\Phi}_{\Omega_{s}}$ measured by the coarse elements is $1.963 \mathrm{~kJ}$. Large warping displacements are visible between the multiple key points and change the void's original geometry from a rectangular shape into a rhombus-like one.

To preserve the outline of the void region and maintain the original distances between the key points, the multi-point shape preserving design [15] methodology is implemented under different shape preserving constraint values and results are listed in Figure 18. 


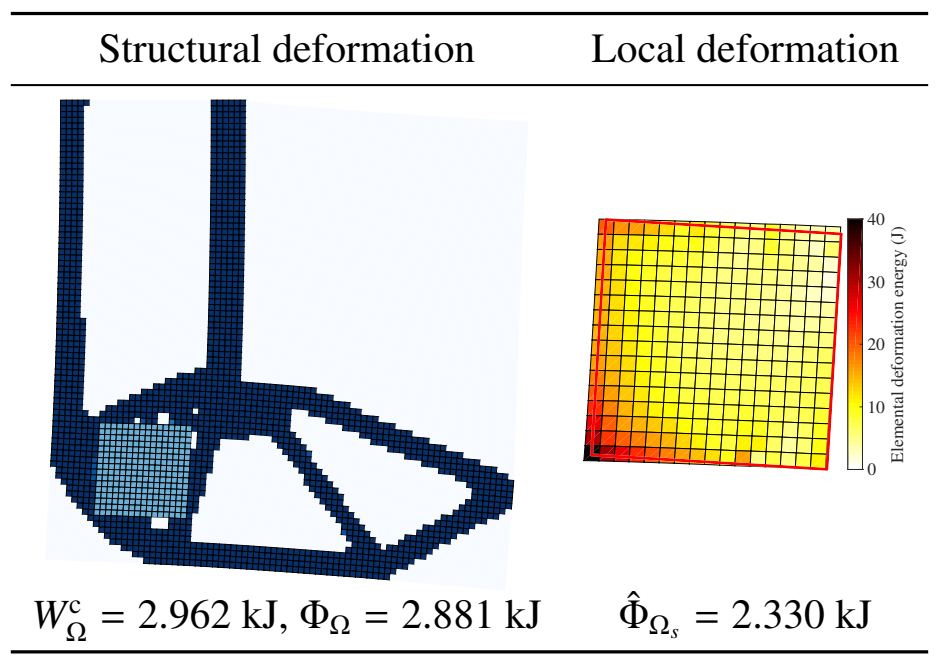

Figure 14: Standard design result of the L-shaped beam with minimized $W_{\Omega}^{\mathrm{c}}$

All the design results satisfy the corresponding warpage control requirement with different material layout patterns. For a constraint value of $30.0 \%$, a great improvement of deformation performance is achieved, where the distortion measurement value of multiple points is now decreased from $1.963 \mathrm{~kJ}$ to $0.367 \mathrm{~kJ}(81.3 \%$ better $)$, with a cost of the structural complementary elastic work increasing from $12.507 \mathrm{~kJ}$ to $13.729 \mathrm{~kJ}$ (7.7\% higher).

For comparison with the multi-point design with a coarse measurement of the integrated deformation energy, another shape preserving design based on the one calculated by the full measurement is included. The design result is shown in Figure 19 under the constraint value of $\varepsilon_{\mathrm{sp}}=30.0 \%$.

A zoom-in on the deformation of the frame is shown in Figure 20. The multi-point design constraint only controls distortions at the key points, while the full measurement approach ensures shape preservation everywhere. Similar differences were also found for the linear case as discussed in [15]. Although the warping deformation inside the shape preserving region is not considered in the multi-point constraint, the internal parts will only be slightly distorted if the outer boundaries are well-preserved.

The outline of the shape preserving domain in the considered cantilever beam problem is very regular, i.e. rectangular. In general, the outline could also be a curved line or have a complex shape. Then the full measurement based on mesh nodes will introduce some difficulties on the boundary calculation and the description of the feature may not be very precise. However, using key points to describe and calculate some primary deformation situations is simpler and more flexible. Another motivation for the coarse measurement is that it is not always possible to establish a full measurement, such as the air inlet outline shown in [15]. There is no void mesh for this spatial profile. Here, the multi-point way based on the boundary key points is very suitable and achievable. 


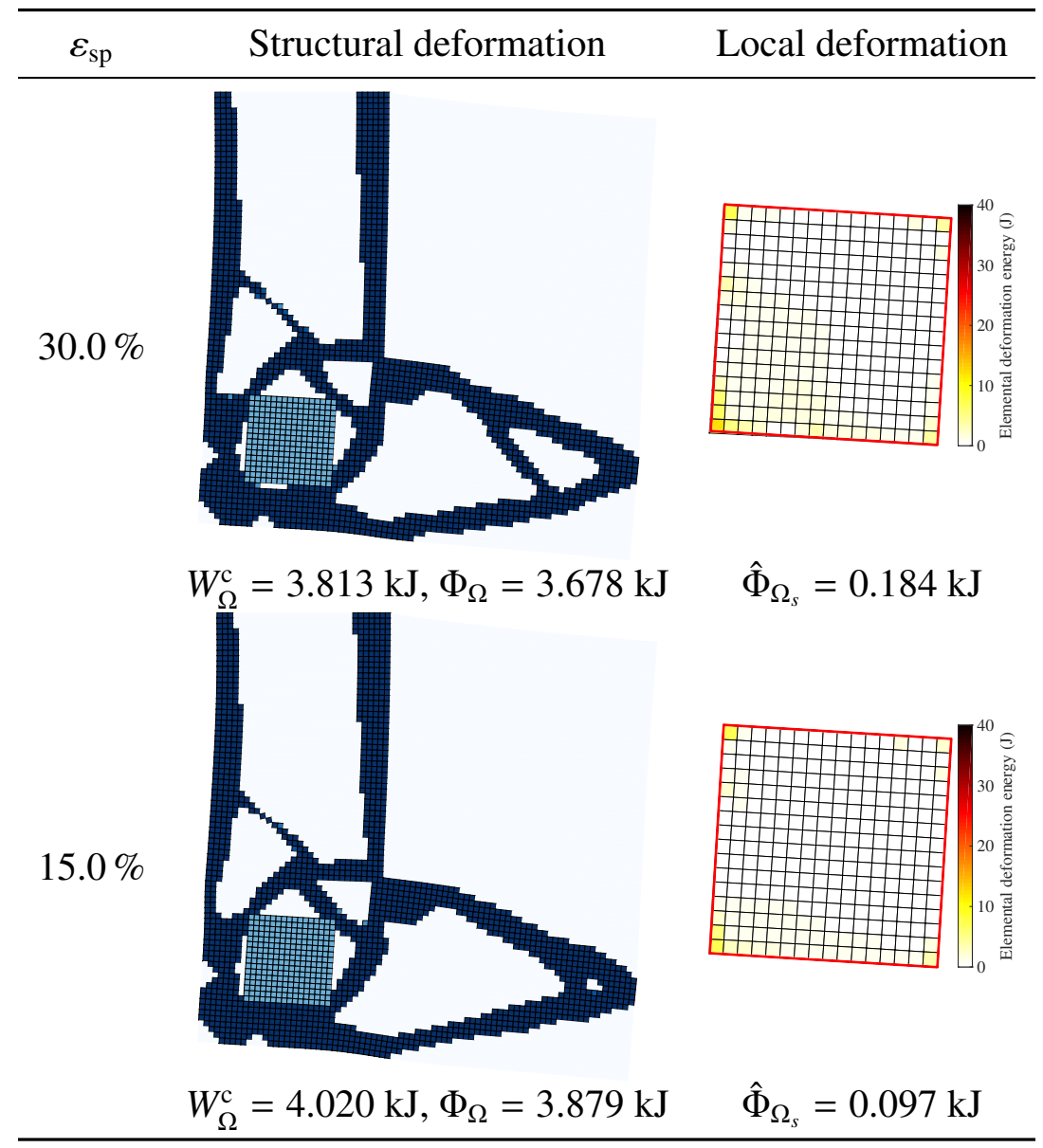

Figure 15: Shape preserving design results for the L-shape beam with minimized $W_{\Omega}^{\mathrm{c}}$ under different constraint values

\subsection{Influence of choice of objective function}

To illustrate the importance of using the integrated complementary work $W_{\Omega}^{\mathrm{c}}$ as opposed to an objective function that only depends on the end load, we include an additional example where we compare a design obtained with the suggested formulation with one optimized for end-compliance $C_{\Omega}$.

The difference is most pronounced for larger loads and hence the same cantilever beam problem as in Sec. 4.1 is used here with a larger load of $230 \mathrm{kN}$. The resulting design, simply minimizing the complementary elastic work, is shown in Figure 21. Subsequently, shape preserving design is carried out with a warpage constraint value of $25 \%$ which is the same as the $25.0 \%$ case in Figure 8. Then, another design minimizing the end-compliance with the same constraint value is run for comparison. Both design results are shown in Figure 22. The design obtained for the complementary elastic work objective results in a reasonable structure as expected, while the end-compliance design generates a hanging rightmost bar, which is vertical in the deformed configuration [18].

Further comparisons of the two designs are based on their force-displacement curves in Figure 23. Here, it is clearly seen that the design obtained for the end-compliance objective has a 


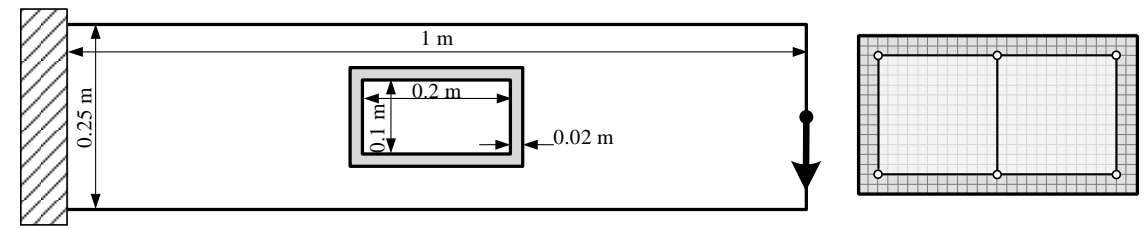

Figure 16: A cantilever beam with a void shape preserving domain and the corresponding coarse measurement of the integrated deformation energy in the shape preserving domain

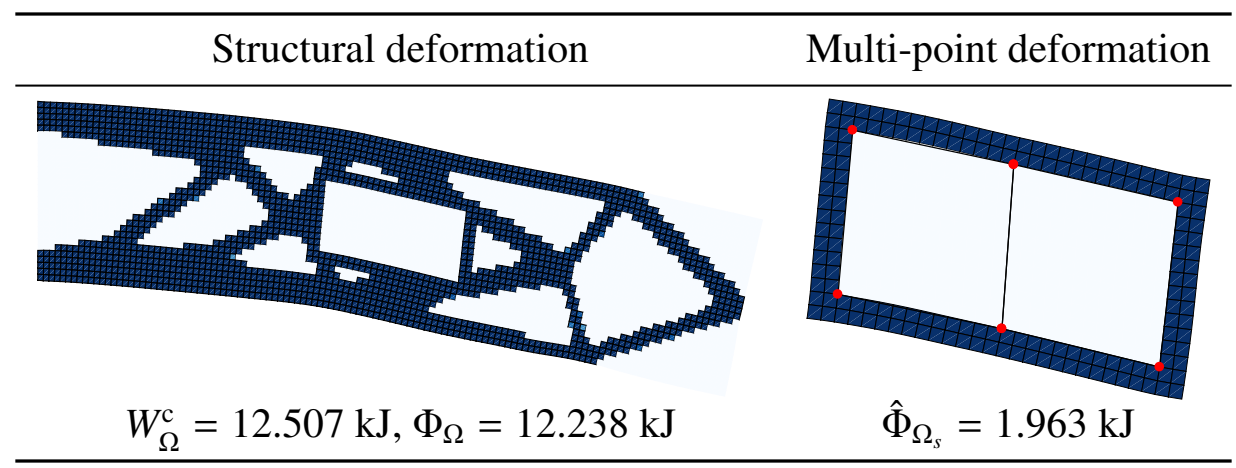

Figure 17: Standard design result for a void subdomain with minimized $W_{\Omega}^{\mathrm{c}}$

smaller deflection at the design load, but larger deformations for smaller loads compared to its integral objective counterpart. Obviously, various design requirements may favor one solution from the other, however, concerning passenger comfort as e.g. in an airplane (and indirectly when considering dynamic effects), it is much preferable to have a structural design solution with a relatively linear load-displacement relationship as seen for the integral objective. This compared to the very large and non-proportional displacements observed for small loads obtained for the end-compliance objective. 


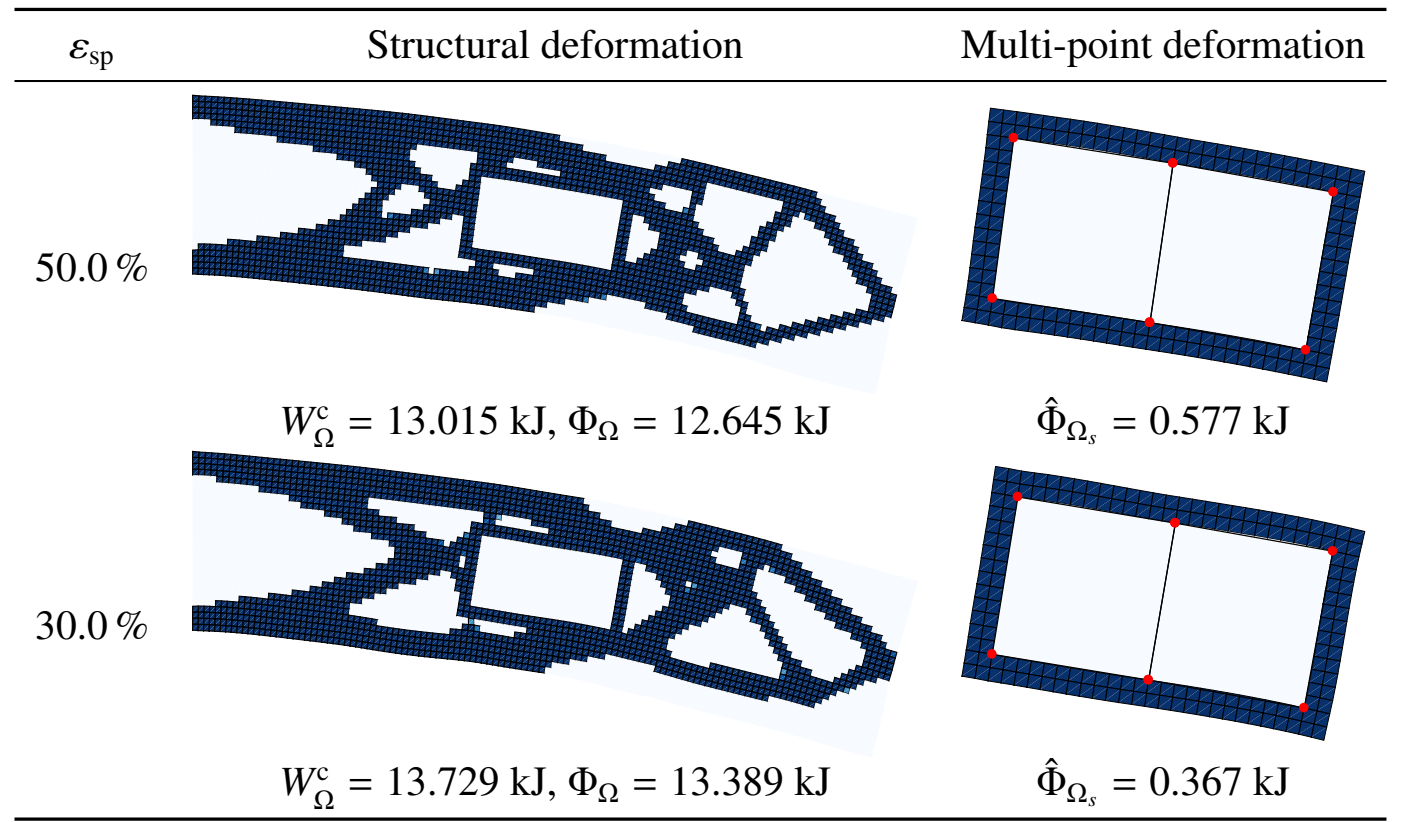

Figure 18: Multi-point shape preserving design results for void subdomain with minimized $W_{\Omega}^{\mathrm{c}}$ under different constraint values

\begin{tabular}{ccc}
\hline$\varepsilon_{\mathrm{sp}}$ & Structural deformation & Local deformation \\
\hline $30.0 \%$ & $W_{\Omega}^{\mathrm{c}}=13.173 \mathrm{~kJ}, \Phi_{\Omega}=12.851 \mathrm{~kJ}$ & $\hat{\Phi}_{\Omega_{s}}=0.352 \mathrm{~kJ}$ \\
\hline
\end{tabular}

Figure 19: Shape preserving design result for a local void domain with minimized $W_{\Omega}^{\mathrm{c}}$ and full distortion measurement 


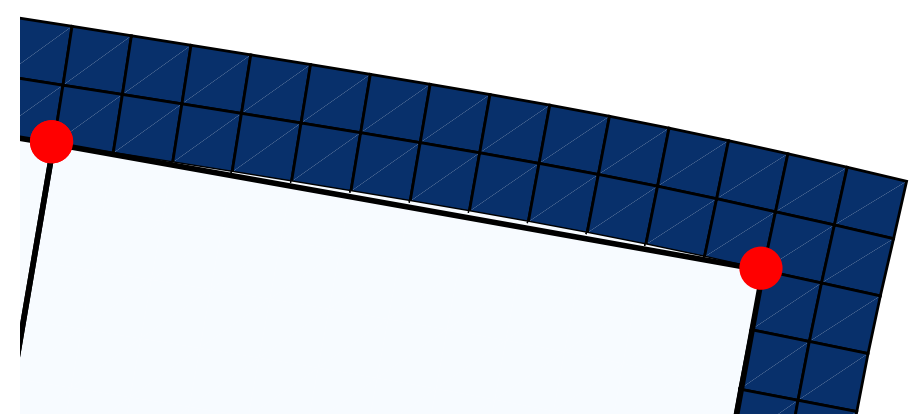

(a) Design result of coarse measurement

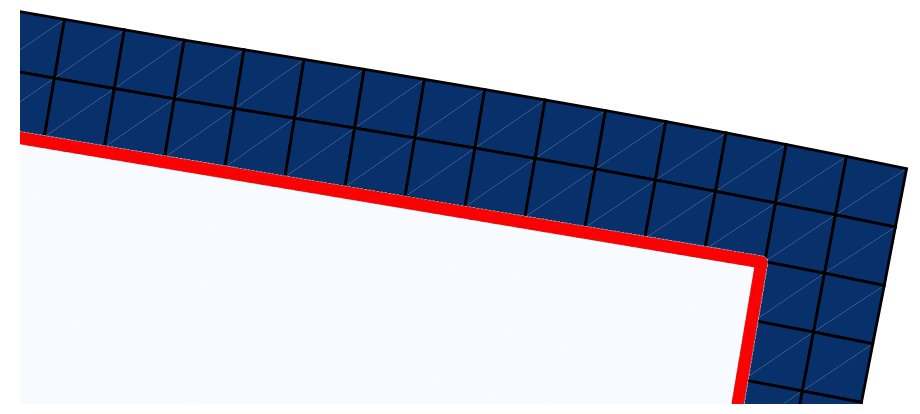

(b) Design result of full measurement

Figure 20: Zoom-in on deformations of the upper right frame region for the designs from Figures 18 and 19

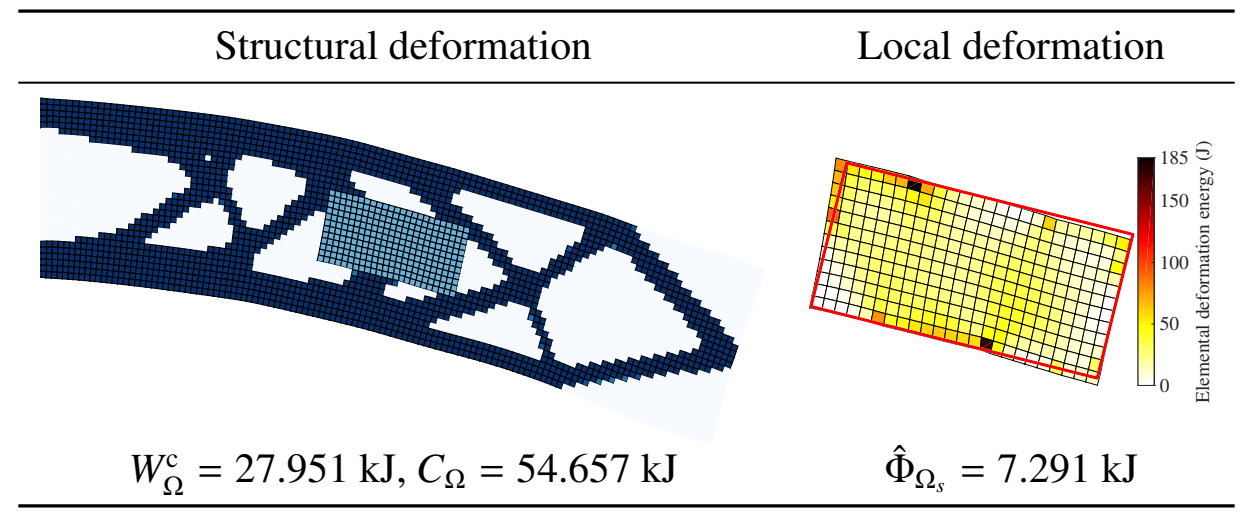

Figure 21: Standard design result for the cantilever beam under $230 \mathrm{kN}$ with minimized $W_{\Omega}^{\mathrm{c}}$ 


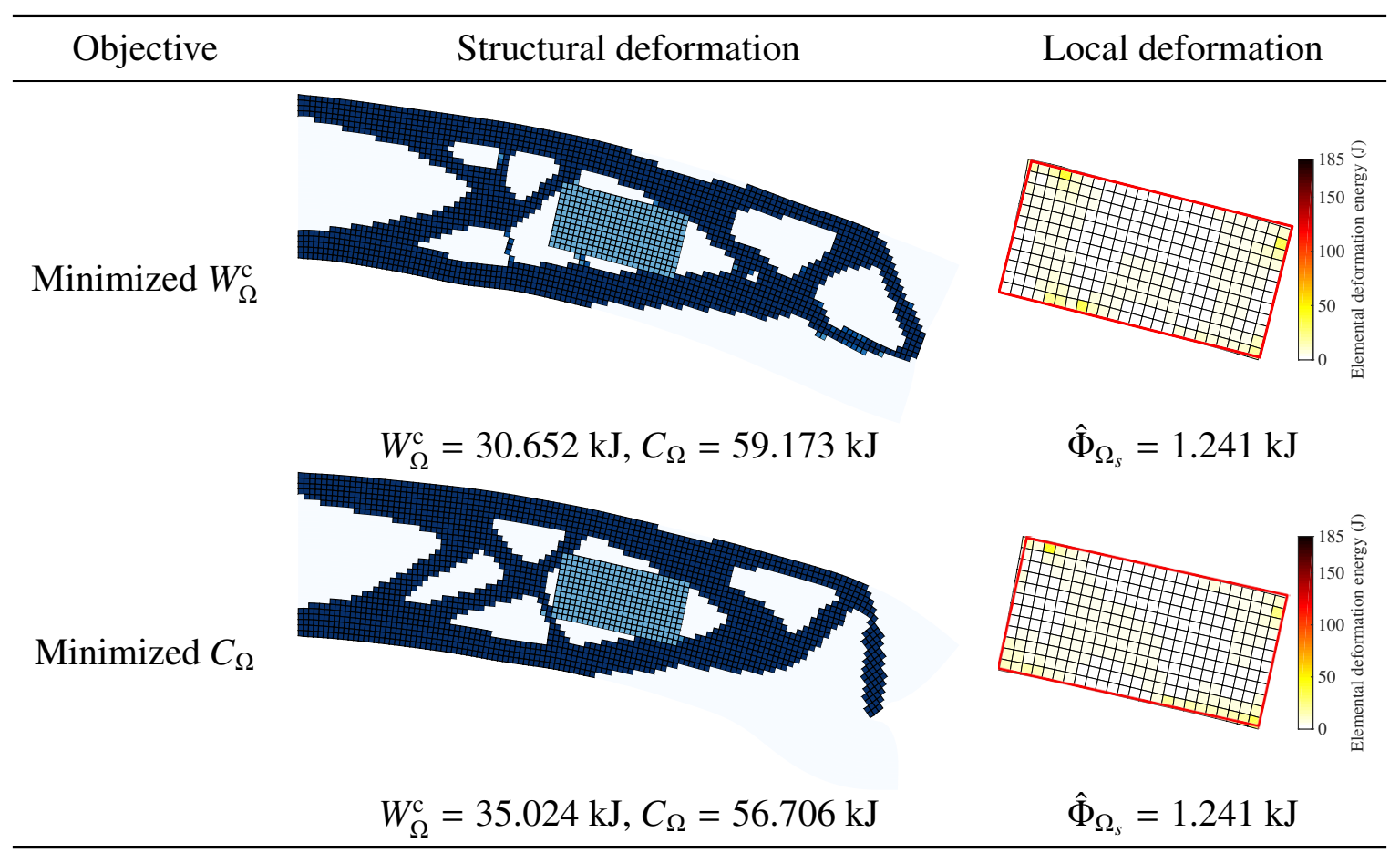

Figure 22: Design results for the cantilever beam with different objective functions

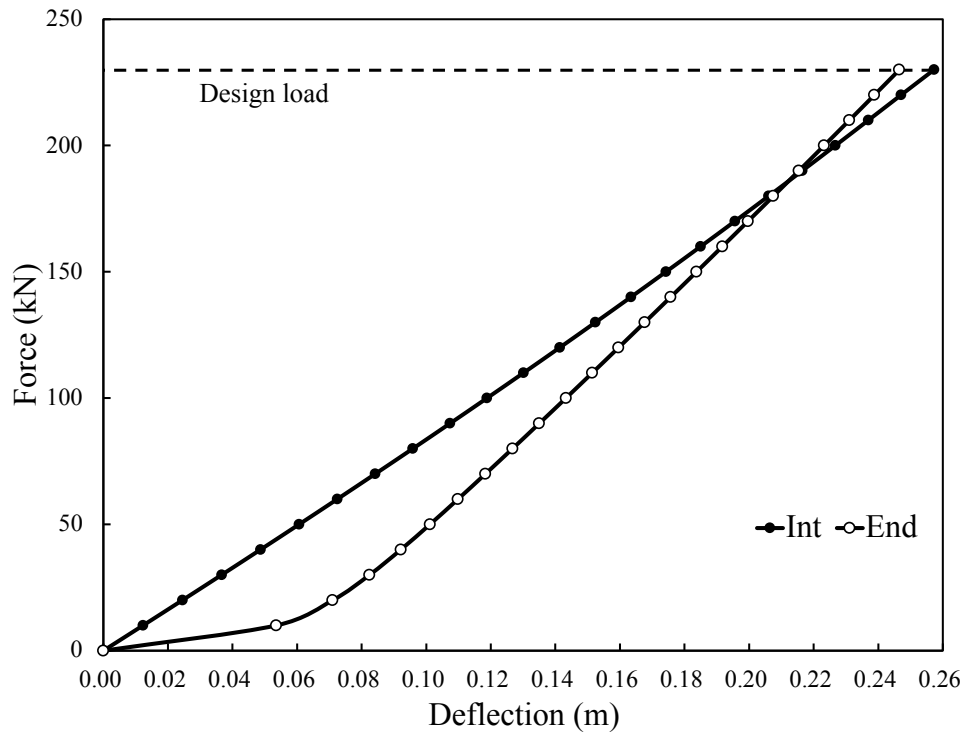

Figure 23: Force-displacement diagram for the design results with different objective functions in Figure 22 (Int: complementary elastic work, End: end-compliance) 


\section{Conclusions}

In this paper, the shape preserving design approach has been extended to the design of geometrically nonlinear structures. Compared to simple linear problems, an improved shape preserving design formulation considering geometrical nonlinearity is proposed to remove the warping deformation in shape preserving domains. Minimum complementary elastic work is assigned as the objective function for stable and practical design results. On the basis of integrated deformation energy, a normalized warpage function is introduced to calculate the geometrical distortion throughout the incremental loading history. The cumulative distortion is constrained to achieve a desirable shape preserving effect, where the considered region moves only in rigid body motion. An additional load case is included to circumvent isolation of the shape preserving domain.

Several numerical examples are tested and parameter studies such as the influence of different constraint values and local stiffnesses as well as the multi-point shape preserving design are carried out. All of the design results clearly demonstrate that warping deformations in shape preserving domains can be effectively suppressed using the suggested setup. Although the approach is validated using only 2D examples based on the Saint Venant-Kirchhoff strain energy density, it is straight forwardly applicable to the $3 \mathrm{D}$ case and alternative hyperelastic constitutive material models with minor modifications. Geometrically nonlinear topology optimization has been performed before without shape preserving constraints in Wang [33]. We remark that the structures presented undergo large displacements, but that strains in solid regions are still relatively small. Hence alternative choices of hyperelastic models are not expected to have a noteworthy influence on optimized device performance for the considered examples but may change algorithmic stability due to deformations in low density regions.

\section{Acknowledgments}

Yu Li would like to acknowledge the financial support from CSC (China Scholarship Council). Fengwen Wang and Ole Sigmund would like to acknowledge the support from the Villum foundation through the VILLUM Investigator project InnoTop. This work is also supported by National Key Research and Development Program (2017YFB1102800), National Natural Science Foundation of China $(11722219,11620101002,51790171,5171101743)$.

\section{Appendix A. Sensitivity analysis of the integrated deformation energy function in shape preserving domains}

From Eq. (11), the integrated deformation energy function in the shape preserving domain is calculated with the trapezoidal method

$$
\begin{aligned}
\Phi_{\Omega_{s}} & \approx \frac{1}{2}\left(\left(\boldsymbol{f}_{\Omega_{s}}^{0}+\boldsymbol{f}_{\Omega_{s}}^{1}\right)^{\mathrm{T}}\left(\boldsymbol{u}_{\Omega_{s}}^{1}-\boldsymbol{u}_{\Omega_{s}}^{0}\right)+\sum_{i=2}^{n-1}\left(\boldsymbol{f}_{\Omega_{s}}^{i-1}+\boldsymbol{f}_{\Omega_{s}}^{i}\right)^{\mathrm{T}}\left(\boldsymbol{u}_{\Omega_{s}}^{i}-\boldsymbol{u}_{\Omega_{s}}^{i-1}\right)+\left(\boldsymbol{f}_{\Omega_{s}}^{n-1}+\boldsymbol{f}_{\Omega_{s}}^{n}\right)^{\mathrm{T}}\left(\boldsymbol{u}_{\Omega_{s}}^{n}-\boldsymbol{u}_{\Omega_{s}}^{n-1}\right)\right) \\
& =\frac{1}{2}\left(\left(\boldsymbol{f}_{\Omega_{s}}^{1^{\mathrm{T}}} \boldsymbol{u}_{\Omega_{s}}^{2}-\boldsymbol{f}_{\Omega_{s}}^{2^{\mathrm{T}}} \boldsymbol{u}_{\Omega_{s}}^{1}\right)+\sum_{i=2}^{n-1}\left(\boldsymbol{f}_{\Omega_{s}}^{i^{\mathrm{T}}} \boldsymbol{u}_{\Omega_{s}}^{i+1}-\boldsymbol{f}_{\Omega_{s}}^{i+1^{\mathrm{T}}} \boldsymbol{u}_{\Omega_{s}}^{i}\right)+\boldsymbol{f}_{\Omega_{s}}^{n^{\mathrm{T}}} \boldsymbol{u}_{\Omega_{s}}^{n}\right)
\end{aligned}
$$


where $\boldsymbol{f}_{\Omega_{s}}^{i}$ and $\boldsymbol{u}_{\Omega_{s}}^{i}$ is the internal force and displacement vector of the shape preserving domain $\Omega_{s}$ in the $i$ th load increment, respectively.

Sensitivity of the integrated deformation energy function in the shape preserving domain with respect to the physical density variable $\bar{\rho}_{e}$ is calculated using adjoint sensitivity analysis. The derivation is further expressed as

$$
\begin{aligned}
\frac{\mathrm{d} \Phi_{\Omega_{s}}}{\mathrm{~d} \bar{\rho}_{e}} & =\sum_{i=1}^{n}\left[\frac{\partial \Phi_{\Omega_{s}}}{\partial \boldsymbol{u}_{i}} \frac{\partial \boldsymbol{u}_{i}}{\partial \bar{\rho}_{e}}+\left(\lambda_{i}\right)^{\mathrm{T}}\left(-\boldsymbol{K}_{i}^{\tan } \frac{\partial \boldsymbol{u}_{i}}{\partial \bar{\rho}_{e}}+\frac{\partial \boldsymbol{r}_{i}}{\partial \bar{\rho}_{e}}\right)\right] \\
& =\sum_{i=1}^{n}\left[\left(\frac{\partial \Phi_{\Omega_{s}}}{\partial \boldsymbol{u}_{i}}-\left(\lambda_{i}\right)^{\mathrm{T}} \boldsymbol{K}_{i}^{\tan }\right) \frac{\partial \boldsymbol{u}_{i}}{\partial \bar{\rho}_{e}}+\left(\lambda_{i}\right)^{\mathrm{T}} \frac{\partial \boldsymbol{r}_{i}}{\partial \bar{\rho}_{e}}\right]
\end{aligned}
$$

where $\boldsymbol{K}_{i}^{\mathrm{tan}}$ is the symmetrical tangent stiffness matrix for increment $i$, and $\partial \boldsymbol{r}_{i} / \partial \boldsymbol{u}_{i}=-\boldsymbol{K}_{i}^{\mathrm{tan}}$. Thus, the corresponding adjoint load for the $i$ th load increment is expressed as

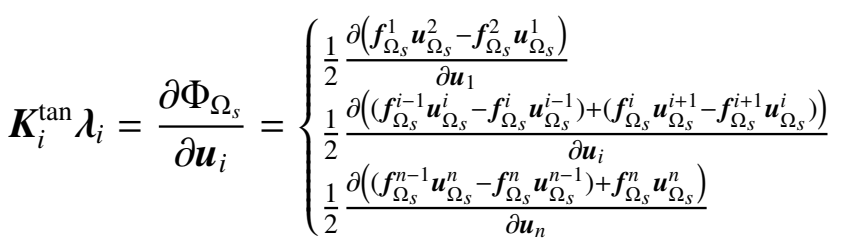

$$
\begin{aligned}
& = \begin{cases}\frac{1}{2}\left(\boldsymbol{K}_{i}^{\tan } \boldsymbol{u}_{\Omega_{s}}^{2}-\boldsymbol{f}_{\Omega_{s}}^{2}\right) & \text { for } i=1 \\
\frac{1}{2}\left(\boldsymbol{K}_{i}^{\tan }\left(\boldsymbol{u}_{\Omega_{s}}^{i+1}-\boldsymbol{u}_{\Omega_{s}}^{i-1}\right)+\left(\boldsymbol{f}_{\Omega_{s}}^{i-1}-\boldsymbol{f}_{\Omega_{s}}^{i+1}\right)\right) & \text { for } i=2 \text { to } n-1 . \\
\frac{1}{2}\left(\boldsymbol{K}_{i}^{\tan }\left(\boldsymbol{u}_{\Omega_{s}}^{n}-\boldsymbol{u}_{\Omega_{s}}^{n-1}\right)+\left(\boldsymbol{f}_{\Omega_{s}}^{n-1}+\boldsymbol{f}_{\Omega_{s}}^{n}\right)\right) & \text { for } i=n\end{cases}
\end{aligned}
$$

By inserting the solutions $\lambda_{i}$ of these linear systems into Eq.(A.2), the final sensitivity is found as

$$
\frac{\mathrm{d} \Phi_{\Omega_{s}}}{\mathrm{~d} \bar{\rho}_{e}}=\sum_{i=1}^{n}\left(\lambda_{i}\right)^{\mathrm{T}} \frac{\partial \boldsymbol{r}_{i}}{\partial \bar{\rho}_{e}} .
$$

\section{References}

[1] O. Sigmund, K. Maute, Topology optimization approaches, Structural and Multidisciplinary Optimization 48 (6) (2013) 1031-1055.

[2] X. Guo, G.-D. Cheng, Recent development in structural design and optimization, Acta Mechanica Sinica 26 (6) (2010) 807-823.

[3] J. D. Deaton, R. V. Grandhi, A survey of structural and multidisciplinary continuum topology optimization: post 2000, Structural and Multidisciplinary Optimization 49 (1) (2014) 1-38.

[4] J.-H. Zhu, W.-H. Zhang, L. Xia, Topology optimization in aircraft and aerospace structures design, Archives of Computational Methods in Engineering 23 (4) (2016) 595-622.

[5] S. Cho, H.-S. Jung, Design sensitivity analysis and topology optimization of displacement-loaded non-linear structures, Computer Methods in Applied Mechanics and Engineering 192 (22) (2003) 2539-2553.

[6] J.-H. Zhu, Y.-B. Zhao, W.-H. Zhang, X.-J. Gu, T. Gao, J. Kong, G.-H. Shi, Y.-J. Xu, D.-L. Quan, Bio-inspired feature-driven topology optimization for rudder structure design, Engineered Sciencedoi : 10.30919/es8d716.

[7] J. Paris, F. Navarrina, I. Colominas, M. Casteleiro, Stress constraints sensitivity analysis in structural topology optimization, Computer Methods in Applied Mechanics and Engineering 199 (33-36) (2010) 2110-2122.

[8] S. Cai, W. Zhang, J. Zhu, T. Gao, Stress constrained shape and topology optimization with fixed mesh: a b-spline finite cell method combined with level set function, Computer Methods in Applied Mechanics and Engineering 278 (2014) 361-387. 
[9] J.-H. Zhu, J. Hou, W.-H. Zhang, Y. Li, Structural topology optimization with constraints on multi-fastener joint loads, Structural and multidisciplinary optimization 50 (4) (2014) 561-571.

[10] T. Gao, L. Qiu, W. Zhang, Topology optimization of continuum structures subjected to the variance constraint of reaction forces, Structural and Multidisciplinary Optimization 56 (4) (2017) 755-765.

[11] L. Krog, A. Tucker, M. Kemp, R. Boyd, Topology optimization of aircraft wing box ribs, in: 10th AIAA/ISSMO multidisciplinary analysis and optimization conference, 2004, pp. 1-11.

[12] N. Aage, E. Andreassen, B. S. Lazarov, O. Sigmund, Giga-voxel computational morphogenesis for structural design., Nature 550 (7674) (2017) 84.

[13] K. Maute, M. Allen, Conceptual design of aeroelastic structures by topology optimization, Structural and Multidisciplinary Optimization 27 (1) (2004) 27-42.

[14] Z. H. Zuo, Y. M. Xie, Evolutionary topology optimization of continuum structures with a global displacement control, Computer-Aided Design 56 (2014) 58-67.

[15] J.-H. Zhu, Y. Li, W.-H. Zhang, J. Hou, Shape preserving design with structural topology optimization, Structural and Multidisciplinary Optimization 53 (4) (2016) 893-906.

[16] Y. Li, J. H. Zhu, W. H. Zhang, L. Wang, Structural topology optimization for directional deformation behavior design with the orthotropic artificial weak element method, Structural and Multidisciplinary Optimization 57 (3) (2017) 1251-1266.

[17] M. S. Castro, O. M. Silva, A. Lenzi, M. M. Neves, Shape preserving design of vibrating structures using topology optimization, Structural and Multidisciplinary Optimization 58 (3) (2018) 1109-1119.

[18] T. Buhl, C. B. Pedersen, O. Sigmund, Stiffness design of geometrically nonlinear structures using topology optimization, Structural and Multidisciplinary Optimization 19 (2) (2000) 93-104.

[19] T. E. Bruns, D. A. Tortorelli, An element removal and reintroduction strategy for the topology optimization of structures and compliant mechanisms, International journal for numerical methods in engineering 57 (10) (2003) 1413-1430.

[20] G. H. Yoon, Y. Y. Kim, Element connectivity parameterization for topology optimization of geometrically nonlinear structures, International journal of solids and structures 42 (7) (2005) 1983-2009.

[21] A. Klarbring, N. Strömberg, Topology optimization of hyperelastic bodies including non-zero prescribed displacements, Structural and Multidisciplinary Optimization 47 (1) (2013) 37-48.

[22] F. Wang, B. S. Lazarov, O. Sigmund, J. S. Jensen, Interpolation scheme for fictitious domain techniques and topology optimization of finite strain elastic problems, Computer Methods in Applied Mechanics and Engineering 276 (2014) 453-472.

[23] Y. Luo, M. Y. Wang, Z. Kang, Topology optimization of geometrically nonlinear structures based on an additive hyperelasticity technique, Computer Methods in Applied Mechanics and Engineering 286 (2015) $422-441$.

[24] P. Pedersen, Elasticity-Anisotropy-Laminates, Department of Mechanical Engineering, Technical University of Denmark, 1998.

[25] M. P. Bendsøe, O. Sigmund, Topology Optimization - Theory, Methods and Applications, Springer Verlag, Berlin Heidelberg, 2004.

[26] O. C. Zienkiewicz, R. L. Taylor, R. L. Taylor, J. Zhu, Finite Element Method: Its Basis and Fundamentals, The: Its Basis and Fundamentals, Elsevier, 2013.

[27] R. Kemmler, A. Lipka, E. Ramm, Large deformations and stability in topology optimization, Structural and Multidisciplinary Optimization 30 (6) (2005) 459-476.

[28] M. Wallin, N. Ivarsson, D. Tortorelli, Stiffness optimization of non-linear elastic structures, Computer Methods in Applied Mechanics and Engineering 330 (2018) 292-307.

[29] E. Andreassen, A. Clausen, M. Schevenels, B. S. Lazarov, O. Sigmund, Efficient topology optimization in matlab using 88 lines of code, Structural and Multidisciplinary Optimization 43 (1) (2011) 1-16.

[30] F. Wang, B. S. Lazarov, O. Sigmund, On projection methods, convergence and robust formulations in topology optimization, Structural and Multidisciplinary Optimization 43 (6) (2011) 767-784.

[31] K. Svanberg, The method of moving asymptotes - a new method for structural optimization, International journal for numerical methods in engineering 24 (2) (1987) 359-373.

[32] R. D. Lahuerta, E. T. Simões, E. M. Campello, P. M. Pimenta, E. C. Silva, Towards the stabilization of the low density elements in topology optimization with large deformation, Computational Mechanics 52 (4) (2013) 
779-797.

[33] F. Wang, Systematic design of 3d auxetic lattice materials with programmable poissons ratio for finite strains, Journal of the Mechanics and Physics of Solids 114 (2018) 303-318. 